Article

\title{
Optional Defaultable Markets
}

\author{
Mohamed N. Abdelghani ${ }^{1, *,+}$ and Alexander V. Melnikov ${ }^{2,+}$ \\ 1 Machine Learning, Morgan Stanley, New York City, NY 10019, USA \\ 2 Mathematical and Statistical Sciences, University of Alberta, Edmonton, AB T6G 2R3, Canada; \\ melnikov@ualberta.ca \\ * Correspondence: mnabdelghani@gmail.com \\ $+\quad$ The research is supported by the NSERC discovery grant 5901.
}

Academic Editor: Mogens Steffensen

Received: 24 September 2017; Accepted: 16 October 2017; Published: 23 October 2017

\begin{abstract}
The paper deals with defaultable markets, one of the main research areas of mathematical finance. It proposes a new approach to the theory of such markets using techniques from the calculus of optional stochastic processes on unusual probability spaces, which was not presented before. The paper is a foundation paper and contains a number of fundamental results on modeling of defaultable markets, pricing and hedging of defaultable claims and results on the probability of default under such conditions. Moreover, several important examples are presented: a new pricing formula for a defaultable bond and a new pricing formula for credit default swap. Furthermore, some results on the absence of arbitrage for markets on unusual probability spaces and markets with default are also provided.
\end{abstract}

Keywords: defaultable claims; hazard process; martingale deflators; optional processes; hedging

\section{Introduction}

Under the usual conditions, a probability space $(\Omega, \mathbf{F}, \mathbf{P})$ is equipped with a non-decreasing family of $\sigma$-algebras $\mathcal{F}_{t} \in \mathbf{F}$ such that $\mathcal{F}_{t}$ is complete and right continuous for all $t$. A wide class of processes adapted to $\mathbf{F}$ are right continuous with left limits paths (RCLL) semimartingales with a well defined stochastic calculus. Many fundamental results in probability theory and mathematical finance were proved with the use of the usual conditions and RCLL semimartingales. However, it is not difficult to give examples showing the existence of a stochastic basis without the usual conditions (see, for instance, Fleming and Harrington (2011, p. 24)). Therefore, in the middle of 1970, the famous expert of stochastic analysis Dellacherie (1975) initiated studies of stochastic processes without this convenient technical assumption. Dellacherie called this case the unusual (nonstandard) conditions. Further developments were done by many mathematicians: Horowitz (1978); Lenglart (1980); Lepingle (1977); Mertens (1972) but mostly by Galchuk $(1980,1982,1985)$. In these publications, a theory of stochastic analysis was constructed for processes on un usual probability spaces. It turns out that many processes on unusual spaces are not necessarily left or right continuous but have left and right limits. For a review of optional calculus and its applications to finance, see Abdelghani (2016); Abdelghani and Melnikov (2016, 2017a, 2017b). A short summary is given in the Appendix A.

Defaultable markets are markets with the possibility of default events occurring. Research on default risk is concerned with modeling of default time, defaultable claims and recovery rules and with pricing and hedging strategies. The two main approaches to modelling default are the structural and the reduced form models. In structural models, the value of the firm determines if a default event occur. This approach was founded by Black and Scholes (1973) and Merton $(1974,1976)$ and extended by many (see Cox and Ross (1976); Geske (1977, 1979); Geske and Johnson (1984); Leland (1994); 
Leland and Toft (1996); Longstaff and Schwartz (1995)). The advantage of structural models is that one sees how the corporate conditions affect default. However, most often the firm's value is not in itself a tradable asset, but the result of an accounting of all of the firm's assets and liabilities where some are visible to the market and others are not. Hence, the parameters of structural models are difficult to estimate in practice. Moreover, in structural models where a boundary condition is used to demarcate the onset of default, it is difficult to define what the boundary condition should be.

In contrast to structural models, in reduced form models, the firm's value plays only an auxiliary role and defaults arrive as a total-surprise to all counterparties. Therefore, default is the result of exogenous factors. In this case, the random time of default is defined as a totally inaccessible stopping time on an enlarged filtration that encompass all market information, assets and defaults. To compute pricing rules and hedging portfolios, a fundamental problem in a reduced form approach is the computation of the conditional probability of default given available market information. To compute the conditional probability of default, several approaches arose depending on whether or not the information about default-free market is available. If default-free market information is not given, then one must suppose the existence of an intensity process associated with the probability of default to be able to price a defaultable claim Duffie and Singleton (1999). This approach is known as the intensity based approach. The main problem with this approach is that the pricing rule is hard to compute Jeanblanc and Yann (2008). On the other hand, in the hazard process approach, the reference filtration of the default-free market is enlarged by the progressive knowledge of default events. It is more convenient to derive probability of default in this framework; however, one needs to assume knowledge of the default-free market information. Much of reduced form pricing theory has been elaborated by many mathematicians (see Artzner and Delbaen (1995); Duffie and Singleton (1997, 1999); Belanger et al. (2001); Elliott et al. (2000); Lando (1998) and Bielecki and Rutkowski (2002), ElKaroui et al. (2010), Jeanblanc (2010), for a good review).

The basic mathematics of structural and reduced form models is given here as a review and, to compare these models to models, we will present in this paper. Let us consider a usual probability space $\left(\Omega, \mathcal{G}, \mathbf{G}=\left(\mathcal{G}_{t}\right)_{t>0}, \mathbf{P}\right)$, where the market evolves: asset, $X$, and default, $\tau$. Associated with default time is the default process of $H_{t}=\mathbf{1}_{(\tau \leq t)}$. Let $\mathcal{F}_{t}$ be the default-free $\sigma$-algebra generated by $X_{t}$ and $\mathcal{H}_{t}$ be the $\sigma$-algebra generated by $H_{t}$ with $\mathbf{F}=\left(\mathcal{F}_{t}\right)_{t \geq 0}$ and $\mathbf{H}=\left(\mathcal{H}_{t}\right)_{t \geq 0}$. Both $\mathcal{F}_{t}$ and $\mathcal{H}_{t}$ are sub- $\sigma$-algebras of $\mathcal{G}_{t}$ for all $t$. In structural form models, the process $X$ is a determinant factor of $\tau$. In other words, the filtrations $\mathcal{F}_{t}$ and $\mathcal{H}_{t}$ may have common elements for any time $t$. In addition, in structural models where default time $\tau$ is predictable, $\mathcal{H}_{t} \subseteq \mathcal{F}_{t}$. On the other hand, in reduced form models, default is not measurable in the reference-filtration $\mathbf{F}$, i.e., $(\tau \leq t) \notin \mathcal{F}_{t}$ for any time $t$. A way to analyze reduced form models is to consider $\tau$ to be an inaccessible stopping time with respect to the market filtration $\mathbf{G}$. With this consideration of $\tau$, one can proceed to compute the conditional probability of default $\mathbf{P}\left(\tau \leq t \mid \mathcal{G}_{t}\right)$ given $\mathcal{G}_{t}$ using intensity-based techniques. If one is able to define $\mathbf{F}$, the default-free filtration, then a new filtration can be constructed by joining the filtration $\mathbf{H}=\left(\mathcal{H}_{t}\right)_{t>0}$ generated by $H_{t}=\mathbf{1}_{(\tau \leq t)}$ to $\mathbf{F}$, i.e., $\tilde{\mathcal{G}}_{t}=\sigma\left(\mathcal{F}_{t} \vee \mathcal{H}_{t}\right) \subseteq \mathcal{G}_{t}$ and $\tilde{\mathbf{G}}=\left(\tilde{\mathcal{G}}_{t}\right)_{t \geq 0}$, then compute $\mathbf{P}\left(\tau \leq t \mid \tilde{\mathcal{G}_{t}}\right)$ using hazard process based techniques. However, this enlargement of the filtration $\mathbf{F}$ by $\mathbf{H}$ leads to several problems in trying to establish a pricing theory based on no-arbitrage principle. Enlargement of filtration changes the properties of martingales and semimartingale. For example, if $X$ is a martingale under $\mathbf{F}$, it might not be a martingale under $\tilde{\mathbf{G}}$ or $\mathbf{G}$. To deal with these effects and to establish existence of local martingale measures for pricing, one has to invoke two invariance principles known as the $\mathbf{H}$ and $\mathbf{H}^{\prime}$ hypotheses. $\mathbf{H}$ hypothesis states that every local martingale in the smaller filtration $\mathcal{F}$ is a local martingale in the larger filtration $\tilde{\mathcal{G}}$, whereas the $\mathbf{H}^{\prime}$ hypothesis states that a semimartingale under the smaller filtration $\mathcal{F}$ remains a semimartingale under the larger filtration $\tilde{\mathcal{G}}$.

While there are still many open and interesting problems to consider in the classical approaches to credit risk, we choose to take another approach to the problem. Our approach is based on the calculus of processes on unusual probability spaces. Even though we are going to use a nonstandard calculus to approach the problem of default, it is possible to view our models as extension or enrichment 
of reduced form models by methods from the calculus of processes on unusual probability spaces. Thus, our goal with this paper is to develop initial results and present applications of the calculus of optional processes to defaultable markets.

However, before we delve into our approach, let us motivate it by few examples where we take a closer look at the mechanics of default and how it affects the value of an asset $X$. Let us place ourselves on the usual probability space $\left(\Omega, \mathcal{F}, \mathbf{F}=\left(\mathcal{F}_{t}\right)_{t \geq 0}, \mathbf{P}\right)$, where the market evolves. In addition, suppose the asset, $X$, remains in existence after default while its value changes. Now, let us fix an instance of time $t$; if default time is predictable or a stopping time in $\mathcal{F}_{t}$, then $(\tau \leq t) \in \mathcal{F}_{t}$-information about default is incorporated in $\mathcal{F}_{t}$. On the other hand, if $(\tau \leq t) \notin \mathcal{F}_{t}$, so that $H_{t}=\mathbf{1}_{(\tau \leq t)}$ is not $\mathcal{F}_{t}$ measurable, then the default time $\tau$ is a random time that is a result of external factors. However, after default takes place, the surprising information about it gets incorporated in future-values of the asset, $X$; if $X_{t}$ is RCLL and $\mathcal{F}_{t}=\mathcal{F}_{t+}$, then obviously $(\tau \leq t) \notin \mathcal{F}_{t+}$ and, loosely speaking, we may want to say $(\tau \leq t) \in \mathcal{F}_{s}$ for any $s>t$ such that $\mathcal{F}_{s} \supset \mathcal{F}_{t+}$ to mean that the information about default time $\tau$ at $t$ is part of a future-filtration of the market. In reduced form modeling, to be precise, an enlarged filtration $\mathbf{F} \vee \mathbf{H}$ is constructed, such that the view that information about default is incorporated in future values of the asset is subsumed in the definition of the enlarged market filtration $\mathbf{F} \vee \mathbf{H}$. However, we are going to show in this paper that, with the use of the calculus of processes on unusual probability spaces, one needs not consider a filtration enlargement to incorporate default information in the dynamic changes of the value of the asset.

Let us consider default from a different viewpoint. Suppose that the event $A \in \mathcal{F}_{\infty}$ such that $\mathbf{P}(A)>0$. Ideally, $\mathcal{F}_{\infty}$ contains all events including defaults, for example, for the default time $\tau$, $\sigma(\tau) \subseteq \mathcal{F}_{\infty}$. The expected value $a_{t}=\mathbf{E}\left[\mathbf{1}_{A} \mid \mathcal{F}_{t}\right]$ at some fixed time, $t$, is implicitly affected by a future default event $\tau$. For example, consider for some fixed time $u$ such that $(2 t<u<\infty)$ and $\tau=u$ where it is possible that $A \cap(\tau=u) \neq \varnothing$. However, at time $t, \mathcal{F}_{t}$ contains absolutely no information about the default event $(\tau=u)$, but the natural filtration $\mathbf{F}^{a}$ generated by the process, $a$, is not default-free. Essentially, in a defaultable market processes that are affected by default at some point over their lifetime, their current value is "implicitly" affected by default even-though current information does not announce the existence of future default events. Therefore, the construction of a defaultable market filtration $\mathbf{G}$ from a default-free filtration $\mathbf{F}$ and a filtration associated with the default process $\mathbf{H}$ is hard to realize in real defaultable markets. This issue can be circumvented by considering defaultable markets on unusual probability spaces.

Let us consider another example. Suppose the asset $X$ that evolves according to $X_{t}^{-1} d X_{t}=\mu d t+\sigma d W_{t}$, where $W$ is a Wiener process and $\mu$ and $\sigma$ are unknown constants to be estimated from market-traded values of $X$. In this market, even though default events might be future events beyond the current market time $t$ are implicit in the $p$ syche of the market, e.g., corporate bonds are riskier than US/Canadian Government bonds, and can weakly influence the current traded values of $X$. As a result, the estimated values of $\mu$ and $\sigma$ from the traded values of $X$ are inadvertently contaminated by default events. Therefore, in defaultable markets, assets and defaults are stitched together and are difficult to segregate.

To avoid difficulties of previous approaches, the reconstruction of enlarged market filtrations, the requiring of invariance properties to be satisfied as a way to get decomposition results, the initial completion of filtration by all null sets from the final filtration, and the requirement that the filtration be right continuous. Without all of these constructs, we propose a different and more natural approach based on the stochastic calculus of optional processes on unusual probability spaces. The paper is organized as follows: Section 2 presents the essence of our approach to defaultable markets on unusual stochastic basis. Section 3 deals with defaultable claims and cash-flows. Section 4 is devoted to the conditional probability of default. In Section 5 , we consider valuation of defaultable contingent claims and illustrative examples. Finally, in Section 6, we discuss the important topic of no-arbitrage condition in markets on unusual spaces. 


\section{Defaultable Markets on Unusual Spaces}

To illustrate our approach, we begin with an example of a simple market that experiences default. Even though simple, the ideas we present here are general and can be carried over to other more delicate market structures. Let us consider now the unusual stochastic basis $\left(\Omega, \mathcal{F}, \mathbf{F}=\left(\mathcal{F}_{t}\right)_{t \geq 0}, \mathbf{P}\right)$ and an asset whose value is given by the process $Y$ on this space. Assume that the asset final value, $\eta$, realized at the end of time is a strictly positive random variable measurable with respect to $\mathcal{F}_{\infty}$. Let $\mathbf{F}=\left(\mathcal{F}_{t}\right)_{t>0}$ represent the history of the market, the good, the bad and defaults. However bad, we assume that the asset never vanquishes, in other words, $Y>0$ for all time. All is well with the market except for a single default event $\tau \in \mathcal{T}\left(\mathbf{F}_{+}\right)$, i.e., $\mathbf{F}_{+}=\left(\mathcal{F}_{t+}\right)_{t \geq 0}$, that happens at some time $t \geq 0$. Notice that we have defined default time, $\tau \in \mathcal{T}\left(\mathbf{F}_{+}\right)$, as an inaccessible stopping time in the broad sense. Furthermore, we assume that the value of the asset before default $\tau$ follows $X$ and immediately after default evolves according to $x$. Both $x$ and $X$ are optional semimartingales adapted to $\mathbf{F}$; however, $X$ is observable only up to default and $x$ is only observable after default. In addition, we define the default process $H=\mathbf{1}_{(\tau<\cdot)}$ as a left continuous optional finite variation process with respect to $\mathbf{F}$. Then, $Y$ is given by

$$
Y_{t}=\left(1-H_{t}\right) X_{t}+H_{t} x_{t}
$$

Note that the filtration generated by $Y$ is the fusion by default of the filtrations generated by $X$ and $x$, which is $\mathcal{F}_{t}^{Y}=\sigma\left(\sigma\left(X_{t \wedge \tau}\right) \vee \sigma\left(x_{t \vee \tau}\right) \vee \sigma\left(H_{t}\right)\right)$. In the integral representation of $Y$, we have,

$$
\begin{aligned}
Y_{t}= & X_{t}-\int_{0+}^{t} H_{s-} d\left(X_{s}-x_{s}\right)-\int_{0}^{t-} H_{s} d\left(X_{s+}-x_{s+}\right) \\
& +\int_{0+}^{t}\left(X_{s-}-x_{s-}\right) d H_{s}+\int_{0}^{t-}\left(X_{s}-x_{s}\right) d H_{s+} \\
& +\int_{0+}^{t} \triangle H_{s} d\left(X_{s}-x_{s}\right)+\int_{0}^{t-} \triangle^{+} H_{s} d\left(X_{s+}-x_{s+}\right) .
\end{aligned}
$$

See Appendix A Equations (A1) and (A2) for the definition of stochastic integrals with respect to optional semimartingales on $u n u$ ual probability space. However, since $H$ has a single left jump but is otherwise continuous, then $\triangle H=0$ and $Y$ reduces to

$$
\begin{aligned}
Y_{t}= & X_{t}-\int_{0+}^{t} H_{s-} d\left(X_{s}-x_{s}\right)-\int_{0}^{t-} H_{s} d\left(X_{s+}-x_{s+}\right) \\
& +\int_{0+}^{t}\left(X_{s-}-x_{s-}\right) H_{s} d s+\int_{0}^{t-}\left(X_{s}-x_{s}\right) H_{s+} d s+\left(X_{\tau+}-x_{\tau+}\right) H_{t} .
\end{aligned}
$$

To highlight the effects of default $\tau$ on the value of $Y$, which will manifest itself as a left jump on the value of $Y$ at the time of default, we are going to assume that $x$ and $X$ are continuous; hence,

$$
Y_{t}=X_{t}-H \circ\left(X_{t}-x_{t}\right)+\left(X_{\tau+}-x_{\tau+}\right) H_{t}+\int_{\tau}^{t}\left(X_{s}-x_{s}\right) d s .
$$

To summarize, the basic tenants to our approach are: (1) the market evolves in the unusual probability space $\left(\Omega, \mathcal{F}, \mathbf{F}=\left(\mathcal{F}_{t}\right)_{t \geq 0}, \mathbf{P}\right) ;(2) \mathbf{F}_{+}$is the smallest right continuous enlargement of $\mathbf{F}$; (3) the value of an asset or a defaultable cash-flow is an F optional semimartingale; and (4) finally, but most importantly, is that default time, $\tau$, is defined as $\tau$ is a totally inaccessible stopping time in the broad sense, i.e., $\tau \in \mathcal{T}\left(\mathbf{F}_{+}\right)$, and the associated default process is $H_{t}=\mathbf{1}_{(\tau<t)}$, which is an optional left continuous process adapted to $\mathcal{F}_{t}$. The definition of default time as an inaccessible stopping time in the broad sense makes default events come as a surprise to the market given up to time $t$ information, $\mathcal{F}_{t}$. However, as the market evolves to $\mathcal{F}_{t+}$, the surprising information about default has been incorporated in $\mathcal{F}_{t+}$. In this way, $\mathcal{F}_{t+}$ in unusual probability space models of defaultable market takes the place of filtration enlargement in reduced form models. Next, we consider defaultable cash-flow pricing and hedging in the context of unusual stochastic basis. 


\section{Defaultable Cash-Flow}

Again, let the market evolve in the unusual probability space $\left(\Omega, \mathcal{F}, \mathbf{F}=\left(\mathcal{F}_{t}\right)_{t>0}, \mathbf{P}\right)$. $\mathbf{P}$ is the real-world probability, as opposed to the local optional martingale measure $\mathbf{Q}$, for which we assume that at least one exists in this market or choose one if many exists Jacod and Protter (2010). In addition, we have $\mathbf{F}_{+}$the smallest right continuous enlargement of $\mathbf{F}$. The filtration $\mathbf{F}$ supports the following objects. The claim, $\Lambda$, is the payoff received by the owner of the claim at maturity time, $T$, if there was no default prior to or at $T$. The process, $A$, with $A_{0}=0$, is the promised dividends if there was no default prior to or at $T$. The recovery claim $\rho$ represents the recovery payoff received at $T$, if default occurs prior to the claims maturity date $T$. The recovery process, $R$, which specifies the recovery payoff at time of default, if it occurs prior to $T$. Finally, default time, $\tau$, is a totally inaccessible stopping time in the broad sense, i.e., $\tau \in \mathcal{T}\left(\mathbf{F}_{+}\right)$. In addition, we define the associated default process by, $H_{t}=\mathbf{1}_{(\tau<t)}$, which is optional and a left continuous process with respect to $\mathcal{F}_{t}$.

Furthermore, we assume that the processes $X, R$, and $A$ are progressively measurable with respect to the filtration $\mathbf{F}$. In addition, the random variable $\Lambda$ is $\mathcal{F}_{T}$-measurable and $\rho$ is at least $\mathcal{F}_{T+}$-measurable. Furthermore, we assume without mentioning that all random objects introduced above are at least RLL and satisfy suitable integrability conditions that are needed for evaluating integrals, stochastic or otherwise. This brings us to the recovery rules; if default occurs after time $T$, then the promised claim $\Lambda$ is paid in full at time $T$. Otherwise, default time $\tau \leq T$ and depending on the agreed upon recovery rules either the amount $R_{\tau}$ is paid at the time of default $\tau$, or the amount $\rho$ is paid at the maturity date $T$. Therefore, in its more general setting, we consider simultaneously both kinds of recovery payoff and thus define a defaultable claim formally as a quintuple, $D C T=(A, \Lambda, \rho, R, H)$. Notice that the date $T$, the information structures $\mathbf{F}$ and $\mathbf{F}_{+}$and the real-world probability $\mathbf{P}$ are intrinsic components of the definition of a defaultable claim (see Bielecki and Rutkowski (2002) for the usual case). For $D C T=(A, \Lambda, \rho, R, H)$, the dividend process is defined as

Definition 1. The dividend process, $D$, of a defaultable claim $D C T=(A, \Lambda, \rho, R, H)$ equals

$$
D_{t}=\tilde{X} \mathbf{1}_{(t \geq T)}+(1-H) \circ A_{t}+R \circ H_{t}
$$

where $\tilde{X}=\left[\Lambda\left(1-H_{T}\right)+\rho H_{T}\right]$. The process $D$ is optional and $\mathbf{F}-$ measurable.

Lemma 1. The process $D$ is finite variation over finite time segments including $[0, T]$.

Proof. By using Galchouk-Ito lemma and properties of the components of $D$.

The risk neutral value of a defaultable claim in this market is the discounted value of the dividend process $D$.

Definition 2. The ex-dividend price process $X(\cdot, T)$ of a defaultable claim $D C T=(A, \Lambda, \rho, R, H)$, which settles at time $T$ is given by

$$
\begin{aligned}
X(t, T) & =B_{t} \mathbf{E}_{\mathbf{Q}}\left(B^{-1} \circ D_{T}-B^{-1} \circ D_{t} \mid \mathcal{F}_{t}\right) \\
& =B_{t} \mathbf{E}_{\mathbf{Q}}\left(\int_{t+}^{T} B_{u-}^{-1} d D_{u}+\int_{t}^{T-} B_{u}^{-1} d D_{u+} \mid \mathcal{F}_{t}\right), \quad \forall t \in[0, T],
\end{aligned}
$$

where $B$ is the discounting process, an optional strictly positive RLL semimartingale.

Under $\mathbf{Q}$, the ex-dividend process, $B_{t}^{-1} X(t, T)$, is a local optional martingale, $B^{-1} X(\cdot, T) \in \mathcal{M}_{l o c}(\mathbf{F}, \mathbf{Q})$, i.e., $B_{s}^{-1} X(s, T)=\mathbf{E}_{\mathbf{Q}}\left[B_{t}^{-1} X(t, T) \mid \mathcal{F}_{s}\right]$ for all $s \leq t$. Expression (3) is referred to as the risk-neutral valuation formula of a defaultable claim (see Musiela and Rutkowski (1997); Duffie and Singleton (1999); Elliott and Kopp (1999) for its definition in usual 
probability spaces). For brevity, write $X_{t}=X(t, T)$ Combine Definitions 1 and 2 and the fact that $H$ is left continuous, we obtain

$$
X_{t}=B_{t} \mathbf{E}_{\mathbf{Q}}\left(B_{T}^{-1} \tilde{X}+\int_{t+}^{T} B_{u-}^{-1}\left(1-H_{u-}\right) d A_{u}+\int_{t}^{T-} B_{u}^{-1}\left(1-H_{u}\right) d A_{u+}+\int_{t}^{T-} B_{u}^{-1} R_{u} d H_{u+} \mid \mathcal{F}_{t}\right) .
$$

Remark 1 (RCLL Cash-Flow). We have considered a general model of defaultable cash-flow, namely that the components of the cash-flow are optional RLL processes affected by default. However, we should not think that we are limited by this generalization of defaultable cash-flow. We can consider that the underlying components of defaultable cash-flow be RCLL processes affected by default, which induces a left continuous optional jump on the resultant defaultable cash-flow, and the theory of defaultable markets on optional space remains the same.

From now on, we are going to work in the deflated probability space $\left(\Omega, \mathcal{F}, \mathbf{F}=\left(\mathcal{F}_{t}\right)_{t \geq 0}, \mathbf{Q}\right)$ and let the expectation operator $\mathbf{E}$ to mean $\mathbf{E}_{\mathbf{Q}}$ - expected values under $\mathbf{Q}$. Next, we provide a justification of definition (3).

\subsection{Portfolio with Default}

Consider a portfolio of three primary securities $S^{2}=B$, the value process of a money market account, $S^{1}$ a default free non-dividend-paying assets, and $S^{0}$ a dividend paying asset, $D$ with $D_{0}=0$, with the possibility of default. Introduce the discounted price processes $\tilde{S}^{i}$ by setting $\tilde{S}_{t}^{i}=S_{t}^{i} / S_{t}^{2}$. The market lifespan is the time interval $[0, \infty]$ and $\phi=\left(\phi^{0}, \phi^{1}, \phi^{2}\right)$ is an F-optional self-financing trading strategy on $\left(S^{0}, S^{1}, S^{2}\right)$ (see Abdelghani and Melnikov (2016) for the definition of optional self-financing trading strategies). It is straightforward to generalize the three-asset portfolio to any number of assets.

To begin with, let us examine a simple trading strategy: suppose that at time $t=0$ we purchase one unit of the $0^{\text {th }}$ asset at the initial price $S_{0}^{0}$ and holds it until time $T$; then, we invest all the proceeds from dividends in the money market account. More specifically, we consider a buy-and-hold strategy $\phi=\left(1,0, \phi^{2}\right)$. The associated wealth process $U$ equals

$$
U_{t}=S_{t}^{0}+\phi_{t}^{2} B_{t}, \quad \forall t \geq 0,
$$

with initial wealth $U_{0}=S_{0}^{0}+\phi_{0}^{2} B_{0}$. Since $\phi$ is self-financing, then

$$
U_{t}-U_{0}=S_{t}^{0}-S_{0}^{0}+\phi^{2} \circ B_{t}+D_{t}
$$

and $B \circ \phi_{t}^{2}+\left[\phi^{2}, B\right]=0$, where $D$ is the dividend paid by $S^{0}$. Now, let us divide $\left(U_{t}-S_{t}^{0}\right)$ by $B$, and use the product rule,

$$
\begin{aligned}
B_{t}^{-1}\left(U_{t}-S_{t}^{0}\right) & =B^{-1} \circ\left(U_{t}-S_{t}^{0}\right)+\left(U_{t}-S_{t}^{0}\right) \circ B_{t}^{-1}+\left[B^{-1},\left(U-S^{0}\right)\right]_{t} \\
& =B^{-1} \circ\left(U_{0}-S_{0}^{0}+\phi^{2} \circ B_{t}+D_{t}\right)+\left(\phi_{t}^{2} B_{t}\right) \circ B_{t}^{-1}+\left[B^{-1}, \phi_{t}^{2} B_{t}\right]_{t} \\
& =\phi^{2} B^{-1} \circ B_{t}+B^{-1} \circ D_{t}+\phi^{2} B \circ B_{t}^{-1}+\left[B^{-1}, \phi^{2} B\right]_{t}
\end{aligned}
$$

where we have used Equations (4) and (5). However, since $B^{-1} \circ B+B \circ B^{-1}=B^{-2} \circ[B, B]$,

$$
\begin{aligned}
{\left[B^{-1}, \phi^{2} B\right] } & =\left[B^{-1}, \phi^{2} \circ B+B \circ \phi^{2}+\left[\phi^{2}, B\right]\right]=\phi^{2} \circ\left[B^{-1}, B\right] \\
& =\phi^{2} \circ\left[-B^{-2} \circ B+B^{-3} \circ[B, B], B\right]=-B^{-2} \phi^{2} \circ[B, B] .
\end{aligned}
$$

Then, we are able to find the simple relation

$$
B_{t}^{-1}\left(U_{t}-S_{t}^{0}\right)=\phi^{2} B_{t}^{-1} \circ B_{t}+\phi^{2} B \circ B_{t}^{-1}-\phi^{2} B^{-2} \circ[B, B]_{t}+B^{-1} \circ D_{t}=B^{-1} \circ D_{t} .
$$


Considering the difference between the value of a defaultable portfolio at time $t$, i.e., $U_{t}$, and its value at maturity $T, U_{T}$, we arrive at the following difference equation:

$$
\begin{gathered}
B_{T}^{-1}\left(U_{T}-S_{T}^{0}\right)-B_{t}^{-1}\left(U_{t}-S_{t}^{0}\right)=B^{-1} \circ D_{T}-B^{-1} \circ D_{t}, \\
B_{T}^{-1} U_{T}-B_{t}^{-1} U_{t}=B_{T}^{-1} S_{T}^{0}-B_{t}^{-1} S_{t}^{0}+B^{-1} \circ D_{T}-B^{-1} \circ D_{t} .
\end{gathered}
$$

Now, we are ready to derive the risk-neutral valuation formula for the ex-dividend price $S_{t}^{0}$. To this end, we have assumed that our model admits a local optional martingale measure $\mathbf{Q}$ equivalent to $\mathbf{P}$ such that the discounted wealth process $B^{-1} U^{\phi}$ of any admissible self-financing trading strategy $\phi$ follow local optional martingales under $\mathbf{Q}$ with respect to the filtration $\mathbf{F}$. Moreover, we make an assumption that the market value at time $t$ of the $0^{\text {th }}$ security comes exclusively from the future dividends stream. This means that we have to postulate that $S_{T}^{0}=0$. This postulate makes sense because the value of a defaultable cash-flow at maturity, which has paid all its value in dividends, is essentially nothing. We shall refer to $S^{0}$ as the ex-dividend price of the $0^{\text {th }}$ asset-the defaultable claim. Given that

$$
\mathbf{E}\left(B_{T}^{-1} U_{T}-B_{t}^{-1} U_{t} \mid \mathcal{F}_{t}\right)=0, \mathbf{E}\left(B_{t}^{-1} S_{t}^{0} \mid \mathcal{F}_{t}\right)=B_{t}^{-1} S_{t}^{0}, S_{T}^{0}=0,
$$

and Equation (6), we arrive at the definition of the value of defaultable claim,

$$
\begin{aligned}
\mathbf{E}\left(B_{T}^{-1} U_{T}-B_{t}^{-1} U_{t} \mid \mathcal{F}_{t}\right) & =\mathbf{E}\left(B_{T}^{-1} S_{T}^{0}-B_{t}^{-1} S_{t}^{0}+B^{-1} \circ D_{T}-B^{-1} \circ D_{t} \mid \mathcal{F}_{t}\right)=0 \Rightarrow \\
B_{t}^{-1} S_{t}^{0} & =\mathbf{E}\left(B^{-1} \circ D_{T}-B^{-1} \circ D_{t} \mid \mathcal{F}_{t}\right) .
\end{aligned}
$$

Hence, $B_{t}^{-1} S_{t}^{0}$ is an $\mathbf{F}$ local optional martingale under $\mathbf{Q}$ and

$$
B_{t}^{-1} S_{t}^{0}=\mathbf{E}\left(B^{-1} \circ D_{T}-B^{-1} \circ D_{t} \mid \mathcal{F}_{t}\right) .
$$

Let us now examine trading with a general self-financing trading strategy $\phi=\left(\phi^{0}, \phi^{1}, \phi^{2}\right)$. The associated wealth process is $U_{t}(\phi)=\sum_{i=0}^{2} \phi_{t}^{i} S_{t}^{i}$. Since $\phi$ is self-financing, then it must be that $U_{t}(\phi)=U_{0}(\phi)+G_{t}(\phi)$ for every $t \geq 0$, where the gains process $G(\phi)$ is

$$
G_{t}(\phi):=\phi^{0} \circ D_{t}+\sum_{i=0}^{2} \phi^{i} \circ S_{t}^{i}=\phi^{0} \circ\left(S_{t}^{0}+D_{t}\right)+\phi^{1} \circ S_{t}^{1}+\phi^{2} \circ S_{t}^{2} .
$$

As before, $S_{t}^{2}=B$, the value of our money market account, $S_{t}^{0}=X(t, T)$ is the dividend, $D$, paying asset and $S^{1}$ is the default-free, non-dividend paying instrument. The term " $\phi^{0} \circ\left(S_{t}^{0}+D_{t}\right)$ " of the gain process is the gain acquired as a result of trading $\phi^{0}$ of the $0^{\text {th }}$ asset having current value $S_{t}^{0}$ and paid dividend $D_{t}$.

Theorem 1. For any self-financing trading strategy, $\phi$, and $\tilde{S}^{i}=B^{-1} S^{i}, i=0,1,2$ are $(\mathbf{F}, \mathbf{Q})$ local optional martingales and the discounted wealth process $B_{t}^{-1} U_{t}(\phi)$ follows a local optional martingale under $(\mathbf{F}, \mathbf{Q})$. 
Proof. Given $\tilde{S}^{i}=B^{-1} S^{i}=S^{i} \circ B^{-1}+B^{-1} \circ S^{i}+\left[B^{-1}, S^{i}\right]$, then, by the product rule we get,

$$
\begin{aligned}
B_{t}^{-1} U_{t}(\phi) & =B_{t}^{-1} \circ U_{t}(\phi)+U_{t}(\phi) \circ B_{t}^{-1}+\left[B^{-1}, U(\phi)\right]_{t} \\
& =B^{-1} \phi^{0} \circ D_{t}+\sum_{i=0}^{2} B^{-1} \phi^{i} \circ S_{t}^{i}+\sum_{i=0}^{2}\left(\phi^{i} S^{i}\right) \circ B_{t}^{-1}+\left[B^{-1}, \sum_{i=0}^{2} \phi^{i} S_{t}^{i}\right] \\
& =B^{-1} \phi^{0} \circ D_{t}+\sum_{i=0}^{2} B^{-1} \phi^{i} \circ S_{t}^{i}+\sum_{i=0}^{2} \phi^{i} S^{i} \circ B_{t}^{-1}+\sum_{i=0}^{2} \phi^{i} \circ\left[B^{-1}, S^{i}\right]_{t} \\
& =\phi^{0} B^{-1} \circ D_{t}+\sum_{i=0}^{2} \phi^{i} B^{-1} \circ S_{t}^{i}+\phi^{i} S^{i} \circ B_{t}^{-1}+\phi^{i} \circ\left[B^{-1}, S^{i}\right]_{t} \\
& =\phi^{0} B^{-1} \circ D_{t}+\sum_{i=0}^{2} \phi^{i} \circ B_{t}^{-1} S_{t}^{i}=\phi^{0} \circ \hat{S}_{t}^{0}+\sum_{i=1}^{2} \phi^{i} \circ \tilde{S}_{t}^{i}
\end{aligned}
$$

where the process $\hat{S}^{0}$ is given by $\hat{S}_{t}^{0}:=\tilde{S}_{t}^{0}+B^{-1} \circ D_{t}$. The process $\hat{S}_{t}^{0}$ is the discounted cumulative dividend price at time $t$ of the defaultable dividend $D$. The processes $\tilde{S}^{i}, i=0,1,2$ are local optional martingales in $(\mathbf{F}, \mathbf{Q})$. To finalize this proof, observe that, using Equation (7), the process $\hat{S}^{0}$ satisfies $\hat{S}_{t}^{0}=\mathbf{E}\left(B^{-1} \circ D_{T} \mid \mathcal{F}_{t}\right)$, and thus follows a local martingale under $\mathbf{Q}$. Hence, $B_{t}^{-1} U_{t}(\phi)$ is a local optional martingale in $(\mathbf{F}, \mathbf{Q})$.

\section{Probability of Default}

The essential component of the defaultable cash-flows, $D$, Equation (2) is the default process $H_{u}=\mathbf{1}_{(\tau<u)}$, where $u \in[0, \infty]$ is a time-horizon. The ex-dividend price process Equation (3) relies implicitly on the conditional expected value of $\mathbf{1}_{(\tau<u)}$ given all known up to time $t$ market information $\mathcal{F}_{t}$. Thus, our goal for this section is to understand the conditional probability of default $F(t, u)=\mathbf{E}\left[H_{u} \mid \mathcal{F}_{t}\right]$, also known as the hazard process and its properties. Associated with the hazard process $F$ is the survival process $G(t, u):=1-F(t, u)$, for which we will discuss some of its properties too. This section is expository, where we will simply list a few interesting related results about $F$ and $G$. We begin by the lemma.

Lemma 2. The process $H_{t}$ is a submartingale.

Proof. Established by conditioning on $\mathcal{F}_{s}$ for any $s \leq t$.

Lemma 3. F and $G$ are positive. Furthermore, for a fixed time horizon, both are optional martingales. However, for a fixed time $t$ and variable time horizon, $F$ is a submartingale and $G$ a supermartingale.

Proof. $F$ and $G$ are positive, and one can write $F(t, u)$

$$
\begin{aligned}
F(t, u) & =\mathbf{E}\left[H_{u} \mid \mathcal{F}_{t}\right]=\mathbf{Q}\left(\tau<u \mid \mathcal{F}_{t}\right)=\mathbf{1}_{(u>t)} \mathbf{E}\left[H_{t}+\mathbf{1}_{(t \leq \tau<u)} \mid \mathcal{F}_{t}\right]+\mathbf{1}_{(u \leq t)} H_{u} \\
& =\mathbf{1}_{(u>t)}\left(H_{t}+\mathbf{E}\left[\left(1-H_{t}\right) H_{u} \mid \mathcal{F}_{t}\right]\right)+\mathbf{1}_{(u \leq t)} H_{u} .
\end{aligned}
$$

For a fixed time horizon $u$ and any $0 \leq s \leq t<u, F(t, u)=\mathbf{E}\left[\mathbf{E}\left[H_{u} \mid \mathcal{F}_{t}\right] \mid \mathcal{F}_{s}\right]=\mathbf{E}\left[H_{u} \mid \mathcal{F}_{s}\right]=F(s, u)$ is a positive optional martingale. In addition, for $0 \leq u \leq s \leq t, F(t, u)=F(s, u)=H_{u}$ a constant. However, for $0 \leq s \leq u \leq t, F(t, u)=\mathbf{E}\left[\mathbf{E}\left[H_{u} \mid \mathcal{F}_{t}\right] \mid \mathcal{F}_{s}\right]=\mathbf{E}\left[H_{u} \mid \mathcal{F}_{s}\right]=F(s, u)$, again, an optional martingale. Essentially for any fixed $u, F(s, u)$ is an optional martingale. The same is true for $G$. For a fixed, $t$, and any time horizons, $0 \leq v \leq u$, where $u$ and $v \in[0, \infty]$. Then, $(\tau<v) \subseteq(\tau<u)$ and

$$
F(t, u)=\mathbf{E}\left[H_{u} \mid \mathcal{F}_{t}\right]=\mathbf{E}\left[H_{v} \mid \mathcal{F}_{t}\right]+\mathbf{E}\left[\mathbf{1}_{(v \leq \tau<u)} \mid \mathcal{F}_{t}\right]=F(t, v)+\mathbf{E}\left[\mathbf{1}_{(v \leq \tau<u)} \mid \mathcal{F}_{t}\right] \geq F(t, v) .
$$


Hence, $F(t, u)$ is increasing along its time horizon. Moreover, for a fixed $t$ and any $v \leq u$,

$$
\begin{aligned}
\mathbf{E}\left(F(t, u) \mid \mathcal{F}_{v}\right) & =\mathbf{E}\left(\mathbf{E}\left(H_{u} \mid \mathcal{F}_{t}\right) \mid \mathcal{F}_{v}\right) \\
& =\mathbf{1}_{(v \geq t)} \mathbf{E}\left(\mathbf{E}\left(H_{v}+\mathbf{1}_{(v \leq \tau<u)} \mid \mathcal{F}_{t}\right) \mid \mathcal{F}_{v}\right)+\mathbf{1}_{(v<t)} \mathbf{E}\left(\mathbf{E}\left(H_{t}+\mathbf{1}_{(t \leq \tau<u)} \mid \mathcal{F}_{t}\right) \mid \mathcal{F}_{v}\right) \\
& =\mathbf{1}_{(v \geq t)} \mathbf{E}\left(H_{v}+\mathbf{1}_{(v \leq \tau<u)} \mid \mathcal{F}_{t}\right)+\mathbf{1}_{(v<t)} \mathbf{E}\left(H_{t}+\mathbf{1}_{(t \leq \tau<u)} \mid \mathcal{F}_{v}\right) \\
& \geq \mathbf{1}_{(v \geq t)} F(t, v)+\mathbf{1}_{(v<t)} F(v, t) .
\end{aligned}
$$

Therefore, if $v \geq t$, then $F(t, u)$ is a submartingale; on the other hand, if $v<t$, then we re-label $v$ as $t$ and $t$ as $v$ to get $\mathbf{E}\left(F(v, u) \mid \mathcal{F}_{t}\right) \geq F(v, t)$, which is also a submartingale. Hence, $F$ is a submartingale in either case. It follows that $G=1-F$ is a supermartingale.

On a related note, Galchuk (1982) showed that for the process $\xi \mathbf{1}_{(\tau<t)}$, where $\xi$ is $\mathcal{F}_{\tau+\text {-measurable }}$ and integrable random variable and $\tau$ is a stopping time in the broad sense, it can be decomposed to an optional martingale that is $\mathcal{F}_{t}$ measurable and an $\mathcal{F}_{t}$ measurable continuous finite variation process. Therefore,

Corollary 1. The default process $H_{t}$ has the following decomposition, $H_{t}=\mathbf{1}_{(\tau<t)}=M_{t}+\mu_{t}$, where $M \in \mathcal{M}(\mathbf{F}, \mathbf{Q})$ is an optional martingale and $\mu$ is the $\mathbf{F}$-compensator of $H$ that is an $\mathbf{F}$-measurable continuous finite variation process.

Proof. A consequence of Theorem 3.5 in Galchuk (1982).

Using the above corollary, let us consider evaluating $\mathbf{E}\left[H_{T} \mid \mathcal{F}_{t}\right]$; for a time horizon $T>t$,

Corollary 2. $F(t, T)=H_{t}+\mathbf{E}\left[\mu_{T}-\mu_{t} \mid \mathcal{F}_{t}\right]$ and $\mathbf{Q}\left(t \leq \tau<T \mid \mathcal{F}_{t}\right)=\mathbf{E}\left[\mu_{T} \mid \mathcal{F}_{t}\right]-\mu_{t}$.

Proof. $F(t, T)=\mathbf{E}\left[\mathbf{1}_{(\tau<T)} \mid \mathcal{F}_{t}\right]$; therefore,

$$
F(t, T)=\mathbf{E}\left[\mathbf{1}_{(\tau<T)} \mid \mathcal{F}_{t}\right]=\mathbf{E}\left[M_{T}+\mu_{T} \mid \mathcal{F}_{t}\right]=M_{t}+\mathbf{E}\left[\mu_{T} \mid \mathcal{F}_{t}\right]=H_{t}+\mathbf{E}\left[\mu_{T}-\mu_{t} \mid \mathcal{F}_{t}\right] .
$$

Consequently, one can write,

$$
\begin{aligned}
\mathbf{Q}\left(t \leq \tau<T \mid \mathcal{F}_{t}\right) & =\left[\left(1-H_{t}\right) H_{T} \mid \mathcal{F}_{t}\right]=\mathbf{E}\left[H_{T}-H_{t} \mid \mathcal{F}_{t}\right]=\mathbf{E}\left[H_{T} \mid \mathcal{F}_{t}\right]-H_{t} \\
& =\mathbf{E}\left[\mu_{T}-\mu_{t} \mid \mathcal{F}_{t}\right]=\mathbf{E}\left[\mu_{T} \mid \mathcal{F}_{t}\right]-\mu_{t} .
\end{aligned}
$$

Let us now understand the relation between the process $\mathbf{1}_{(\tau \leq t)}$, for a stopping time in the broad sense $\tau$, and the default process $H_{t}=\mathbf{1}_{(\tau<t)}$ as regards the filtration $\mathbf{F}$.

Remark 2. Recall that, under the usual conditions, $\mathbf{1}_{(\tau \leq t)}$ is known as the default process; however, in the unusual conditions, it is replaced with the $\mathcal{O}(\mathbf{F})$ process $H_{t}=\mathbf{1}_{(\tau<t)}$. This choice of $\mathbf{1}_{(\tau<t)}$ as the default process was motivated by the fact that the known market information up to time $t$ is $\mathcal{F}_{t}$ on which $\mathbf{1}_{(\tau<t)}$ is adapted and optional while $\mathbf{1}_{(\tau \leq t)}$ is not. Furthermore, the forward derivative $\triangle^{+} H_{t}=\mathbf{1}_{(\tau=t)}$ marks the occurrence of default, which is in $\mathcal{O}\left(\mathbf{F}_{+}\right)$.

Proposition 1. Let $\tau$ be a stopping time in the broad sense and $\bar{H}_{t}=H_{t+}=\mathbf{1}_{(\tau \leq t)}$, where $(\tau \leq t)=(\tau<t+)$, is RCLL and $\mathcal{F}_{t+}$ measurable or $\mathcal{O}\left(\mathbf{F}_{+}\right)$. In addition, $\bar{H}_{t-}=H_{t}$ or $(\tau \leq t-)=(\tau<t)$. $\bar{H}_{t-}$ and $H_{t}$ are of right-limits and left continuous paths and are $\mathcal{O}(\mathbf{F})$. Moreover, $(\tau \leq t)=(\tau<t) \cup(\tau=t)$ or that $\bar{H}_{t}=H_{t}+\mathbf{1}_{(\tau=t)}$. 
Proof. For every $\omega \in(\tau \leq t), \tau(\omega) \leq t<t+$ so $(\tau \leq t) \subseteq(\tau<t+)$. On the other hand, $(\tau(\omega)<t+)=\cap_{\epsilon \geq 0}(\tau<t+\epsilon) \subseteq(\tau \leq t)$. Thus, $H_{t+}=\mathbf{1}_{(\tau<t+)}=\mathbf{1}_{(\tau \leq t)}$ or that $(\tau \leq t)=(\tau<t+)$. By definition, $\mathbf{1}_{(\tau \leq t)}$ is RCLL and $\mathcal{O}\left(\mathbf{F}_{+}\right)$. Furthermore, it can be easily seen that the process $\mathbf{1}_{(\tau \leq t)}$ can be decomposed as, $\mathbf{1}_{\tau \in[0, t]}=\mathbf{1}_{\tau \in[0, t)}+\mathbf{1}_{(\tau=t)}$. In addition, it is obvious that $\bar{H}_{t-}=\mathbf{1}_{(\tau \leq t-)}=$ $\mathbf{1}_{(\tau<t)}=H_{t}$. Finally, for $\tau$ a stopping time in the broad sense, by definition, $(\tau \leq t) \in \mathcal{F}_{t+}$ for any time $t$. Moreover, since $(\tau \leq t-1 / n) \in \mathcal{F}_{(t-1 / n)+} \subseteq \mathcal{F}_{t}$ for all $n$, then it must be that $(\tau<t) \in \mathcal{F}_{t}$.

Let us study the properties of the process $F(t, t+)=\mathbf{E}\left(H_{t+} \mid \mathcal{F}_{t}\right)=\mathbf{Q}\left(\tau \leq t \mid \mathcal{F}_{t}\right)$. Set $\bar{F}_{t}:=\mathbf{E}\left(H_{t+} \mid \mathcal{F}_{t}\right)$ and consider the following lemma.

Lemma 4. The ex-hazard process $\bar{F}$ can be decomposed to the default process $H$ and the jump hazard process $\delta$. Both $\bar{F}$ and $\delta$ are $\mathcal{O}\left(\mathbf{F}_{+}\right)$, whereas $H$ is $\mathcal{O}(\mathbf{F})$.

Proof. The optional projection $\mathbf{E}\left(H_{t+} \mid \mathcal{F}_{t}\right)$ can be reduced to

$$
\mathbf{E}\left(H_{t+} \mid \mathcal{F}_{t}\right)=\mathbf{E}\left(\mathbf{1}_{(\tau \leq t)} \mid \mathcal{F}_{t}\right)=\mathbf{E}\left(\mathbf{1}_{(\tau<t)}+\mathbf{1}_{(\tau=t)} \mid \mathcal{F}_{t}\right)=H_{t}+\mathbf{E}\left(\mathbf{1}_{(\tau=t)}\right)=H_{t}+\delta_{t}
$$

where $\delta_{t}$ can be derived by formula, $\delta_{t}=\lim _{h \rightarrow 0} \frac{1}{h} \mathbf{Q}\left(t \leq \tau<t+h \mid \mathcal{F}_{t}\right)$.

Corollary 3. $F(t, T+)=F(t, T)+\mathbf{E}\left(\delta_{T} \mid \mathcal{F}_{t}\right)=M_{t}+\mathbf{E}\left(\mu_{T}+\delta_{T} \mid \mathcal{F}_{t}\right)$.

Proof. The process $F(t, T+)=\mathbf{E}\left(H_{T+} \mid \mathcal{F}_{t}\right)$ is evaluated as

$$
\begin{gathered}
F(t, T+)=\mathbf{E}\left(H_{T} \mid \mathcal{F}_{t}\right)+\mathbf{E}\left(\mathbf{1}_{\tau=T} \mid \mathcal{F}_{t}\right)=H_{t}+\mathbf{E}\left[\mu_{T}-\mu_{t} \mid \mathcal{F}_{t}\right]+\mathbf{E}\left(\delta_{T} \mid \mathcal{F}_{t}\right)=F(t, T)+\mathbf{E}\left(\delta_{T} \mid \mathcal{F}_{t}\right) \\
\text { or } F(t, T+)=H_{t}-\mu_{t}+\mathbf{E}\left(\mu_{T}+\delta_{T} \mid \mathcal{F}_{t}\right)=M_{t}+\mathbf{E}\left(\mu_{T}+\delta_{T} \mid \mathcal{F}_{t}\right) .
\end{gathered}
$$

Next, we discuss the absence of arbitrage in markets on unusual spaces.

\section{Valuation of Defaultable Cash-Flow and Examples}

Our next goal is to establish a convenient representation of the value of a defaultable cash-flow. The ex-dividend value of defaultable cash-flow is $B_{t}^{-1} X(t, T)=\mathbf{1}_{(\tau \geq t)} \mathbf{E}\left(B^{-1} \circ D_{T}-B^{-1} \circ D_{t} \mid \mathcal{F}_{t}\right)$, and, in terms of $D_{t}=\left(\Lambda\left(1-H_{T}\right)+\rho H_{T}\right) \mathbf{1}_{(t \geq T)}+(1-H) \circ A_{t}+R \circ H_{t}$, is

$$
\begin{aligned}
B_{t}^{-1} X_{t}= & \mathbf{E}\left[B_{T}^{-1}\left(\Lambda\left(1-H_{T}\right)+\rho H_{T}\right) \mid \mathcal{F}_{t}\right] \\
& +\mathbf{E}\left[\int_{t+}^{T} B_{u-}^{-1}\left(1-H_{u-}\right) d A_{u}+\int_{t}^{T-} B_{u}^{-1}\left(1-H_{u}\right) d A_{u+} \mid \mathcal{F}_{t}\right] \\
& +\mathbf{E}\left[\int_{t}^{T-} B_{u}^{-1} R_{u} d H_{u+} \mid \mathcal{F}_{t}\right] .
\end{aligned}
$$

We begin valuation of $X(t, T)$ with the value of $\mathbf{E}\left[B_{T}^{-1} \Lambda\left(1-H_{T}\right) \mid \mathcal{F}_{t}\right]$. Let $\lambda_{T}=B_{T}^{-1} \Lambda$ and $\lambda_{t}=\mathbf{E}\left(\lambda_{T} \mid \mathcal{F}_{t}\right)$ be a martingale. $\lambda_{t}$ can be thought of as the default-free contingent claim price at time $t$. Let $G(t, u)=\mathbf{E}\left(1-H_{u} \mid \mathcal{F}_{t}\right)$. $G$ can be thought of as the survival process, whereas $F(t, u)=\mathbf{E}\left(H_{u} \mid \mathcal{F}_{t}\right)$ is the associated default process on the interval $[t, u]$.

Lemma 5. The value of $\tilde{\Lambda}_{t}=\mathbf{E}\left[B_{T}^{-1} \Lambda\left(1-H_{T}\right) \mid \mathcal{F}_{t}\right]$ at time $t$ is given by

$$
\tilde{\Lambda}_{t}=\mathbf{E}\left(B_{T}^{-1} \Lambda \mid \mathcal{F}_{t}\right)\left(1-H_{t}\right)+\mathbf{E}\left[\int_{t}^{T-}\left(\lambda_{u}+\Delta^{+} \lambda_{u}\right) d G_{u+} \mid \mathcal{F}_{t}\right],
$$

where $\lambda_{u}=\mathbf{E}\left[B_{T}^{-1} \Lambda \mid \mathcal{F}_{u}\right]$ and $G_{u+}=G(u, u+)=\mathbf{E}\left(1-H_{u+} \mid \mathcal{F}_{u}\right)$. 
Proof. Using the product rule on $\lambda_{T}\left(1-H_{T}\right)$, we find that

$$
\begin{aligned}
\lambda_{T}\left(1-H_{T}\right)= & \lambda_{0}\left(1-H_{0}\right)+\lambda \circ\left(1-H_{T}\right)+G \circ \lambda_{T}+[\lambda,(1-H)]_{T} \\
= & \lambda_{0}\left(1-H_{0}\right)+\lambda \circ\left(1-H_{t}\right)+G \circ \lambda_{t}+[\lambda,(1-H)]_{t}+\int_{t+}^{T} \lambda_{u-} d(1-H)_{u} \\
& +\int_{t}^{T-} \lambda_{u} d(1-H)_{u+}+\int_{t+}^{T}(1-H)_{u-} d \lambda_{u}+\int_{t}^{T-}(1-H)_{u} d \lambda_{u+} \\
& +\int_{t}^{T-} \triangle^{+} \lambda_{u} d(1-H)_{u+} \\
= & \lambda_{t}(1-H)_{t}+\int_{t}^{T} \lambda_{u} d(1-H)_{u}+\int_{t}^{T}(1-H)_{u} d \lambda_{u}+\int_{t}^{T-} \triangle^{+} \lambda_{u} d(1-H)_{u+} .
\end{aligned}
$$

Since $H$ is a left continuous finite variation process, the quadratic variation

$$
[\lambda, H]_{t}=\sum_{0 \leq u<t} \triangle^{+} \lambda_{u} \triangle^{+} H_{u+}=\int_{0}^{t-} \triangle^{+} H_{u} d \lambda_{u+}=\int_{0}^{t-} \triangle^{+} \lambda_{u} d H_{u+}
$$

We have chosen the definition $[\lambda, H]=\triangle^{+} \lambda \odot H$. Thus, the conditional expected value of $\lambda_{T}\left(1-H_{T}\right)$ is $\mathbf{E}\left(\lambda_{T}\left(1-H_{T}\right) \mid \mathcal{F}_{t}\right)=\mathbf{E}\left(B_{T}^{-1} \Lambda\left(1-H_{T}\right) \mid \mathcal{F}_{t}\right)$ and

$$
\begin{aligned}
\mathbf{E}\left(\lambda_{T}\left(1-H_{T}\right) \mid \mathcal{F}_{t}\right) & =\mathbf{E}\left[\lambda_{t}\left(1-H_{t}\right)+\int_{t}^{T}\left(1-H_{u}\right) d \lambda_{u}+\int_{t}^{T-} \lambda_{u} d\left(1-H_{u+}\right)+\int_{t}^{T-} \triangle^{+} \lambda_{u} d\left(1-H_{u+}\right) \mid \mathcal{F}_{t}\right] \\
& =\lambda_{t}\left(1-H_{t}\right)+\mathbf{E}\left[\int_{t}^{T-}\left(\lambda_{u}+\triangle^{+} \lambda_{u}\right) d G_{u+} \mid \mathcal{F}_{t}\right]
\end{aligned}
$$

where we have used the fact that $\mathbf{E}\left[\int_{t}^{T} G_{u} d \lambda_{u} \mid \mathcal{F}_{t}\right]=0$ since $\lambda$ is a local martingale, $G_{u+}=G(u, u+)$ and $\mathbf{E}\left(\lambda_{t}\left(1-H_{t}\right) \mid \mathcal{F}_{t}\right)=\lambda_{t}\left(1-H_{t}\right)$. Thus, we arrive at the result

$$
\mathbf{E}\left[B_{T}^{-1} \Lambda\left(1-H_{T}\right) \mid \mathcal{F}_{t}\right]=\mathbf{E}\left(B_{T}^{-1} \Lambda \mid \mathcal{F}_{t}\right)\left(1-H_{t}\right)+\mathbf{E}\left[\int_{t}^{T-}\left[\mathbf{E}\left(B_{T}^{-1} \Lambda \mid \mathcal{F}_{u}\right)+\triangle^{+} \mathbf{E}\left(B_{T}^{-1} \Lambda \mid \mathcal{F}_{u}\right)\right] d G_{u+} \mid \mathcal{F}_{t}\right] .
$$

Remark 3. We have replaced the integral over $1-H$ with an integral over the process $G$ as a result of the following statement:

$$
\begin{aligned}
\mathbf{E}\left[\int_{t}^{T-} \alpha_{u} d H_{u+} \mid \mathcal{F}_{t}\right] & \leftarrow \sum_{i=0}^{n} \mathbf{E}\left[\alpha_{t_{i}} \mathbf{E}\left(H_{t_{i+1} \wedge T}-H_{t_{i} \wedge T} \mid \mathcal{F}_{t_{i}}\right) \mid \mathcal{F}_{t}\right] \\
& =\sum_{i=0}^{n} \mathbf{E}\left[\alpha_{t_{i}} F_{t_{i+1} \wedge T}-F_{t_{i} \wedge T} \mid \mathcal{F}_{t}\right]=\mathbf{E}\left[\left(\sum_{i=0}^{n} \alpha_{t_{i}} F_{t_{i+1} \wedge T}-F_{t_{i} \wedge T}\right) \mid \mathcal{F}_{t}\right]
\end{aligned}
$$

where $F_{u+}=F(u, u+)=\mathbf{E}\left(H_{u+} \mid \mathcal{F}_{u}\right)$.

Remark 4. How do we evaluate $d G_{u+}$ ? Here is how: $G(u, u+)=\mathbf{E}\left(1-H_{u+} \mid \mathcal{F}_{u}\right)$ and $d G_{u+}=\triangle^{+} G_{u}=G(u, u+)-G(u, u)$. Therefore,

$$
d G_{u+}=\mathbf{E}\left(1-H_{u+} \mid \mathcal{F}_{u}\right)-\mathbf{E}\left(1-H_{u} \mid \mathcal{F}_{u}\right)=\mathbf{E}\left(H_{u}-H_{u+} \mid \mathcal{F}_{u}\right),
$$

where $H_{u+}=\mathbf{1}_{(\tau \leq u)}=H_{u}+\mathbf{1}_{(\tau=u)}$. Hence, $d G_{u+}=-\mathbf{E}\left(\mathbf{1}_{(\tau=u)} \mid \mathcal{F}_{u}\right)=-\mathbf{Q}\left(\tau=u \mid \mathcal{F}_{u}\right)$. Note that the event $(\tau=u)$ is $\mathcal{F}_{u+}$ measurable so $d G_{u+}$ is the projection of $(\tau=u)$ on $\mathcal{F}_{u}$.

For the second term, $\mathbf{E}\left(B_{T}^{-1} \rho H_{T} \mid \mathcal{F}_{t}\right)$ : let $\varrho_{T}=B_{T}^{-1} \rho$ thus $\varrho_{t}=\mathbf{E}\left(\varrho_{T} \mid \mathcal{F}_{t}\right)$ is a local martingale. In addition, we have the following lemma. 
Lemma 6. The value $\tilde{\rho}_{t}=\mathbf{E}\left(\varrho_{T} H_{T} \mid \mathcal{F}_{t}\right)$ is given by

$$
\tilde{\rho}_{t}=\varrho_{t} H_{t}-\mathbf{E}\left[\int_{t}^{T-}\left(\varrho_{u}+\Delta^{+} \varrho_{u}\right) d G_{u+} \mid \mathcal{F}_{t}\right],
$$

where $\varrho_{u}=\mathbf{E}\left(B_{T}^{-1} \rho \mid \mathcal{F}_{u}\right)$.

Proof. Applying a similar argument to the one used in the above lemma, we arrive at the result

$$
\begin{aligned}
\tilde{\rho}_{t} & =\mathbf{E}\left(\varrho_{T} H_{T} \mid \mathcal{F}_{t}\right)=\mathbf{E}\left[\varrho_{t} H_{t}+\int_{t}^{T} H_{u} d \varrho_{u}+\int_{t}^{T} \varrho_{u} d H_{u}+\int_{t}^{T-} \Delta^{+} \varrho_{u} d H_{u+} \mid \mathcal{F}_{t}\right] \\
& =\varrho_{t} H_{t}+\mathbf{E}\left[\int_{t}^{T-}\left(\varrho_{u}+\triangle^{+} \varrho_{u}\right) d F_{u+} \mid \mathcal{F}_{t}\right] \\
& =\varrho_{t} H_{t}-\mathbf{E}\left[\int_{t}^{T-}\left(\varrho_{u}+\triangle^{+} \varrho_{u}\right) d G_{u+} \mid \mathcal{F}_{t}\right],
\end{aligned}
$$

where $\mathbf{E}\left[\int_{t}^{T} H_{u} d \varrho_{u} \mid \mathcal{F}_{t}\right]=0$ since $\varrho$ is a local martingale.

As for the value of the defaultable cash-flow recovery-stream, we have the following lemma.

Lemma 7. The value of the defaultable cash-flow recovery stream is

$$
\mathbf{E}\left[\int_{t}^{T-} B_{u}^{-1} R_{u} d H_{u+} \mid \mathcal{F}_{t}\right]=-\mathbf{E}\left[\int_{t}^{T-} B_{u}^{-1} R_{u} d G_{u+} \mid \mathcal{F}_{t}\right] .
$$

Proof. Using the sum approximation of the left optional integral, the ex-dividend value of defaultable claim is derived as follows:

$$
\begin{aligned}
\mathbf{E}\left[\int_{t}^{T-} B_{u}^{-1} R_{u} d H_{u+} \mid \mathcal{F}_{t}\right] & =\mathbf{E}\left[\int_{t}^{T-} \mathbf{E}\left[B_{u}^{-1} R_{u} d H_{u+} \mid \mathcal{F}_{u}\right] \mid \mathcal{F}_{t}\right]=\mathbf{E}\left[\int_{t}^{T-} B_{u}^{-1} R_{u} d F_{u+} \mid \mathcal{F}_{t}\right] \\
& =-\mathbf{E}\left[\int_{t}^{T-} B_{u}^{-1} R_{u} d G_{u+} \mid \mathcal{F}_{t}\right]
\end{aligned}
$$

Note that $B_{u}^{-1} R_{u}$ is $\mathcal{F}_{u}$ measurable.

Finally, we look at the process $\mathbf{E}\left[B^{-1}(1-H) \circ A_{T}-B^{-1}(1-H) \circ A_{t} \mid \mathcal{F}_{t}\right]$. Let $\hat{A}_{t}=B^{-1} \circ$ $A_{t}$; then,

$$
\begin{aligned}
\mathbf{E}\left[B^{-1}(1-H) \circ A_{T}-B^{-1}(1-H) \circ A_{t} \mid \mathcal{F}_{t}\right] & =\mathbf{E}\left[(1-H) \circ \hat{A}_{T}-(1-H) \circ \hat{A}_{t} \mid \mathcal{F}_{t}\right] \\
& =\mathbf{E}\left[\int_{t}^{T}\left(1-H_{u}\right) d \hat{A}_{u} \mid \mathcal{F}_{t}\right]
\end{aligned}
$$

Now, let us apply the product rule to $\left(1-H_{t}\right) \hat{A}_{t}$ from $[t, T]$,

$$
\left(1-H_{T}\right) \hat{A}_{T}=\left(1-H_{t}\right) \hat{A}_{t}+\int_{t}^{T} \hat{A} d(1-H)_{u}+\int_{t}^{T}(1-H) d \hat{A}_{u}+\int_{t}^{T} \triangle^{+} \hat{A}_{u} d(1-H) .
$$

Using the above equation, we can write

$$
\int_{t}^{T}(1-H) d \hat{A}_{u}=\left(1-H_{T}\right) \hat{A}_{T}-\left(1-H_{t}\right) \hat{A}_{t}-\int_{t}^{T-} \hat{A}_{u} d(1-H)_{u}-\int_{t}^{T-} \triangle^{+} \hat{A}_{u} d(1-H)_{u+} .
$$

Without loss of generality, we are going to assume that $A_{T}=0$ because any dividend payment made at time $T$ is going to be part of the claim $\Lambda$ paid at maturity time $T$. Hence, the equation above reduces to 


$$
\int_{t}^{T}(1-H) d \hat{A}_{u}=-\left(1-H_{t}\right) \hat{A}_{t}-\int_{t}^{T-} \hat{A}_{u} d(1-H)_{u+}-\int_{t}^{T-} \triangle^{+} \hat{A}_{u} d(1-H)_{u+} .
$$

We are going to use this equation to prove the next lemma.

Lemma 8. The value of dividend payout stream is

$$
\begin{aligned}
\mathbf{E}\left[B^{-1}(1-H) \circ A_{T}-B^{-1}(1-H) \circ A_{t} \mid \mathcal{F}_{t}\right]= & -\left(1-H_{t}\right)\left(B^{-1} \circ A_{t}\right) \\
& -\mathbf{E}\left[\int_{t}^{T-}\left(\left(B^{-1} \circ A_{u}\right)+\Delta^{+}\left(B^{-1} \circ A_{u}\right)\right) d G_{u+} \mid \mathcal{F}_{t}\right] .
\end{aligned}
$$

Proof. Using Equation (9), the sum approximation of the left optional integral and the fact that $\left(1-H_{t}\right)\left(B^{-1} \circ A_{t}\right)$ is $\mathcal{F}_{t}$ measurable, we get

$$
\begin{aligned}
\mathbf{E}\left[B^{-1}(1-H) \circ A_{T}-B^{-1}(1-H) \circ A_{t} \mid \mathcal{F}_{t}\right]= & \mathbf{E}\left[\int_{t}^{T}(1-H) d \hat{A}_{u} \mid \mathcal{F}_{t}\right] \\
= & \mathbf{E}\left[-\left(1-H_{t}\right) \hat{A}_{t}-\int_{t}^{T-} \hat{A}_{u} d(1-H)_{u+} \mid \mathcal{F}_{t}\right] \\
& -\mathbf{E}\left[\int_{t}^{T-} \triangle^{+} \hat{A}_{u} d(1-H)_{u+} \mid \mathcal{F}_{t}\right] \\
= & -\left(1-H_{t}\right) \hat{A}_{t} \\
& -\mathbf{E}\left[\int_{t}^{T-}\left(\hat{A}_{u}+\Delta^{+} \hat{A}_{u}\right) d(1-H)_{u+} \mid \mathcal{F}_{t}\right] \\
= & -\left(1-H_{t}\right)\left(B^{-1} \circ A_{t}\right) \\
& -\mathbf{E}\left[\int_{t}^{T-}\left(\left(B^{-1} \circ A_{u}\right)+\Delta^{+}\left(B^{-1} \circ A_{u}\right)\right) d G_{u+} \mid \mathcal{F}_{t}\right],
\end{aligned}
$$

where $\hat{A}=B^{-1} \circ A$.

Consequently, we arrive at the following theorem for the ex-dividend price process.

Theorem 2. The ex-dividend price process $X(\cdot, T)$ that settles at time $T$ is

$$
\begin{aligned}
B_{t}^{-1} X(t, T)= & \lambda_{t}\left(1-H_{t}\right)+\mathbf{E}\left[\int_{t}^{T-}\left(\lambda_{u}+\Delta^{+} \lambda_{u}\right) d G_{u+} \mid \mathcal{F}_{t}\right] \\
& +\varrho_{t} H_{t}-\mathbf{E}\left[\int_{t}^{T-}\left(\varrho_{u}+\Delta^{+} \varrho_{u}\right) d G_{u+} \mid \mathcal{F}_{t}\right] \\
& -\left(1-H_{t}\right) \hat{A}_{t}-\mathbf{E}\left[\int_{t}^{T-}\left(\hat{A}_{u}+\Delta^{+} \hat{A}_{u}\right) d G_{u+} \mid \mathcal{F}_{t}\right] \\
& -\mathbf{E}\left[\int_{t}^{T-} \hat{R}_{u} d G_{u+} \mid \mathcal{F}_{t}\right] \\
= & \left(\lambda_{t}-\hat{A}_{t}\right)\left(1-H_{t}\right)+\varrho_{t} H_{t} \\
& +\mathbf{E}\left[\int_{t}^{T-}\left(\lambda_{u}-\varrho_{u}-\hat{A}_{u}-\hat{R}_{u}+\Delta^{+}\left(\lambda_{u}-\varrho_{u}-\hat{A}_{u}\right)\right) d G_{u+} \mid \mathcal{F}_{t}\right],
\end{aligned}
$$

where $\lambda_{t}=\mathbf{E}\left(B_{T}^{-1} \Lambda \mid \mathcal{F}_{t}\right), \varrho_{t}=\mathbf{E}\left(B_{T}^{-1} \rho \mid \mathcal{F}_{t}\right), \hat{A}_{t}=B^{-1} \circ A_{t}$ and $\hat{R}_{t}=B_{t}^{-1} R_{t}$.

Proof. Follows from the lemmas above.

In the special case where components of the dividends process are RCLL processes, we get the following corollary.

Corollary 4. Suppose we are given a defaultable cash-flow $A$, and $R$ and $B$ are RCLL. Then, the ex-dividend price process $X(\cdot, T)$ that settles at time $T$ is

$$
\begin{aligned}
X(t, T)= & B_{t}\left(\lambda_{t}-\hat{A}_{t}\right)\left(1-H_{t}\right)+B_{t} \varrho_{t} H_{t} \\
& +B_{t} \mathbf{E}\left[\int_{t}^{T-}\left(\lambda_{u}-\varrho_{u}-\hat{A}_{u}-\hat{R}_{u}+\Delta^{+}\left(\lambda_{u}-\varrho_{u}\right)\right) d G_{u+} \mid \mathcal{F}_{t}\right] .
\end{aligned}
$$


Furthermore, if $\lambda_{t}=\mathbf{E}\left(B_{T}^{-1} \Lambda \mid \mathcal{F}_{t}\right)$ and $\varrho_{t}=\mathbf{E}\left(B_{T}^{-1} \rho \mid \mathcal{F}_{t}\right)$ have RCLL modifications, then $\triangle^{+} \mathbf{E}\left(B_{T}^{-1} \Lambda \mid \mathcal{F}_{t}\right)=0$ and $\triangle^{+} \mathbf{E}\left(B_{T}^{-1} \rho \mid \mathcal{F}_{t}\right)=0$ for all $t$ and

$$
\begin{aligned}
X(t, T)= & B_{t}\left(\lambda_{t}-\hat{A}_{t}\right)\left(1-H_{t}\right)+B_{t} \varrho_{t} H_{t} \\
& +B_{t} \mathbf{E}\left[\int_{t}^{T-}\left(\lambda_{u}-\varrho_{u}-\hat{A}_{u}-\hat{R}_{u}\right) d G_{u+} \mid \mathcal{F}_{t}\right] .
\end{aligned}
$$

Proof. Follows from the definition of the ex-dividend price process.

We were able to establish a convenient representation of the value of a defaultable claim in terms of the probability of default. Now, we provide some examples.

Example 1 (Zero-Coupon Defaultable Bond). The price of a zero-coupon bond with face-value $\$ 1$ at maturity date $T$ is $B_{t} \mathbf{E}\left(B_{T}^{-1} \mid \mathcal{F}_{t}\right)$ at time $t$. On the other hand, the price of a zero-coupon bond that may experience default is $B_{t} \mathbf{E}\left(B_{T}^{-1} \mathbf{1}_{(\tau \geq T)} \mid \mathcal{F}_{t}\right)$. Lemma 5 tells us how to compute the price $B_{t} \mathbf{E}\left(B_{T}^{-1} \mathbf{1}_{(\tau \geq T)} \mid \mathcal{F}_{t}\right)$ at time $t$. Let $\lambda_{T}=B_{T}^{-1}$; thus, $\lambda_{t}=\mathbf{E}\left(\lambda_{T} \mid \mathcal{F}_{t}\right)$ and

$$
\begin{aligned}
B_{t}^{-1} X(t, T) & =\mathbf{E}\left(\lambda_{T}\left(1-H_{T}\right) \mid \mathcal{F}_{t}\right) \\
& =\lambda_{t}\left(1-H_{t}\right)+\mathbf{E}\left[\int_{t}^{T-}\left(\lambda_{u}+\Delta^{+} \lambda_{u}\right) d G_{u+} \mid \mathcal{F}_{t}\right] \\
& =\lambda_{t} \mathbf{1}_{(\tau \geq t)}+\mathbf{E}\left[\int_{t}^{T-}\left(\lambda_{u}+\triangle^{+} \lambda_{u}\right) d G_{u+} \mid \mathcal{F}_{t}\right] .
\end{aligned}
$$

Suppose that $B_{t}=e^{r t}$, where $B(t, T)=B_{t} B_{T}^{-1}=e^{-r(T-t)}$, with a constant interest rate $r$ and that the survival process admits a constant intensity $\gamma$ such that

$$
\begin{aligned}
d G_{u+} & =\mathbf{E}\left(H_{u}-H_{u+} \mid \mathcal{F}_{u}\right)=-\mathbf{E}\left(\mathbf{1}_{(\tau=u)} \mid \mathcal{F}_{u}\right) \\
& =-\delta(u-\tau) \gamma e^{-\gamma u} d u,
\end{aligned}
$$

where $\delta(u-\tau)$ is Dirac delta function at a particular value of default time $\tau$. Then,

$$
\begin{aligned}
X(t, T) & =\mathbf{1}_{(\tau \geq t)} e^{-r(T-t)}-e^{-r(T-t)} \mathbf{E}\left[\int_{t}^{T} \delta(u-\tau) \gamma e^{-\gamma u} d u \mid \mathcal{F}_{t}\right] \\
& =e^{-r(T-t)}\left[\mathbf{1}_{(\tau \geq t)}-\mathbf{1}_{(t \leq \tau<T)} e^{-\gamma t}\left(1-e^{-\gamma(T-t)}\right)\right]
\end{aligned}
$$

At $t=0, X(0, T)=e^{-r T}\left[1-\mathbf{1}_{(0 \leq \tau<T)}\left(1-e^{-\gamma T}\right)\right]$, and, at $t=T, X(T, T)=\mathbf{1}_{(\tau \geq T)}$. If $\tau<T$, then

$$
X(0, T)=e^{-(r+\gamma) T} .
$$

Therefore, for a bond of face-value of $\$ 1$ at maturity date $T$, default decreases the present value of the bond by a factor $e^{-\gamma T}$. Hence, $X(t, T)$ is a reasonable pricing formula. In addition, note that, in this simple case, the price formula we presented here matches the price formula derived with classical methods in the usual case (see the following references for comparison Duffie and Singleton (2012, p. 102); Bingham and Kiesel (2013, p. 396); Martellini et al. (2003, p. 448)).

Example 2 (Credit Default Swap). A credit default swap (CDS) is a contract in which the holder of a defaultable asset buys an insurance against default. The elements of a CDS are: the maturity time $T$, the fee rate function $K(t)$ and the recovery function $R(t)$. The buyer of default protection pays a fee at a rate $K$ up to default 
time $\tau$ or to maturity time $T$ and receives the amount $R(\tau)$ at default from the seller of the protection. The price of the CDS at time $t$ is given by the difference in price between the value of the protective leg and premium leg:

$$
C D S(t, T)=\operatorname{Prot}(t, T)-\operatorname{Prem}(t, T),
$$

where the protection price is

$$
\begin{aligned}
\operatorname{Prot}(t, T) & =\mathbf{E}\left(R(\tau) \mathbf{1}_{t \leq \tau<T} \mid \mathcal{F}_{t}\right)=\mathbf{E}\left(\int_{t}^{T-} R_{u} d H_{u+} \mid \mathcal{F}_{t}\right) \\
& =\mathbf{E}\left(\int_{t}^{T-} R_{u} d F_{u+} \mid \mathcal{F}_{t}\right)
\end{aligned}
$$

and the premium price is

$$
\operatorname{Prem}(t, T)=\mathbf{E}\left(\mathbf{1}_{t \leq \tau} \int_{t}^{\tau \wedge T} K(u) d u \mid \mathcal{F}_{t}\right)=\mathbf{E}\left(\int_{t}^{T}\left(1-H_{u}\right) K_{u} d u \mid \mathcal{F}_{t}\right)
$$

Suppose $K$ and $R$ are constants and default admits a constant intensity $\gamma$ such that

$$
\begin{aligned}
d F_{u+} & =\mathbf{E}\left(H_{u+}-H_{u} \mid \mathcal{F}_{u}\right)=\mathbf{E}\left(\mathbf{1}_{(\tau=u)} \mid \mathcal{F}_{u}\right) \\
& =\delta(u-\tau) \gamma e^{-\gamma u} d u,
\end{aligned}
$$

at a particular value of default time $\tau$. Then, the legs prices are

$$
\begin{aligned}
\operatorname{Prot}(t, T) & =\mathbf{E}\left(\int_{t}^{T-} R_{u} d F_{u+} \mid \mathcal{F}_{t}\right)=R \int_{t}^{T-} \delta(u-\tau) \gamma e^{-\gamma u} d u \\
& =\mathbf{1}_{(t \leq \tau<T)} R e^{-\gamma t}\left(1-e^{-\gamma(T-t)}\right),
\end{aligned}
$$

and

$$
\begin{aligned}
\operatorname{Prem}(t, T) & =\mathbf{E}\left(\int_{t}^{T}\left(1-H_{u}\right) K_{u} d u \mid \mathcal{F}_{t}\right)=K \mathbf{E}\left(\int_{t}^{T}\left(1-F_{u}\right) d u \mid \mathcal{F}_{t}\right) \\
& =K \mathbf{E}\left(\int_{t}^{T} \delta(u-\tau) e^{-\gamma u} d u \mid \mathcal{F}_{t}\right)=\mathbf{1}_{(t \leq \tau<T)} \frac{K}{\gamma} e^{-\gamma t}\left(1-e^{-\gamma(T-t)}\right)
\end{aligned}
$$

Therefore, $\operatorname{CDS}(t, T)=\left(R-\gamma^{-1} K\right)\left(1-e^{-\gamma(T-t)}\right) e^{-\gamma t} \mathbf{1}_{(t \leq \tau<T)}$. At $t=0$,

$$
\operatorname{CDS}(0, T)=\left(R-\gamma^{-1} K\right)\left(1-e^{-\gamma T}\right) \mathbf{1}_{(\tau<T)} .
$$

\section{Absence of Arbitrage on Unusual Spaces}

The no-arbitrage arguments culminated in the fundamental theorem of asset pricing: under the usual conditions, for a real valued semimartingale $X$, there exists a probability measure $\mathbf{Q}$ equivalent to $\mathbf{P}$ under which $X$ is a $\sigma$-martingale if and only if $X$ does not permit a free lunch with vanishing risk. Given that $K=\left\{(\phi \cdot X)_{\infty}: \phi\right.$ admissible and $(\phi \cdot X)_{\infty}=\lim _{t \rightarrow \infty}(\phi \cdot X)_{t}$ exists a.s. $\}$ and $C=\left\{g \in L^{\infty}(\mathbf{P})\right.$ : $g \leq f \forall f \in K\}$, then $X$ is said to satisfy no free lunch with vanishing risk (NFLVR) if $\bar{C} \cap L_{+}^{\infty}(\mathbf{P})=\{0\}$, where $\bar{C}$ is the closure of $C$ in the norm topology of $L_{+}^{\infty}(\mathbf{P})$ Delbaen and Schachermayer (2006). $\mathbf{Q}$ is known as the equivalent local martingale measure (ELMM). However, in some models of financial markets, ELMM may fail to exist. An alternative was developed, an equivalent local martingale deflator (ELMD), which is a strictly positive local martingale that transforms the semimartingale $X$ to a local martingale. It was shown that the existence of a strictly positive local martingale deflator is equivalent to the no arbitrage condition of the first kind (NA1). An $\mathcal{F}_{T}$ measurable random variable $\zeta$ 
is called an arbitrage of the first kind if $\mathbf{P}(\zeta \geq 0)=1, \mathbf{P}(\zeta>0)>0$ and for any initial wealth $x>0$ if there exists an admissible $\phi$ such that $x+\phi \cdot X_{T} \geq \zeta$ Kardaras (2012). NA1 is a weaker condition than NFLVR. While extending NFLVR and NA1 and the equivalence relations ELMM and ELMD, respectively, to unusual stochastic basis is possible and is of importance, it is definitely out of scope here. However, here we will present an argument to show the viability of financial markets and markets with defaults on unusual probability spaces.

Again, consider a market on unusual probability space $\left(\Omega, \mathcal{F}, \mathbf{F}=\left(\mathcal{F}_{t}\right)_{t \geq 0}, \mathbf{P}\right)$. Introduce the filtration $\mathbf{F}_{+}=\left(\mathcal{F}_{t+}\right)_{t \geq 0}$ and its completion under $\mathbf{P}, \tilde{\mathbf{F}}=\left(\mathcal{F}_{t+}^{\mathbf{P}}\right)_{t \geq 0}$. In addition, let $Y \in \mathcal{O}(\mathbf{F})$ be a real valued optional semimartingale that is at least of RLL paths and $\tilde{Y}=\left(Y_{t+}\right)_{t \geq 0}$ is $\mathcal{O}(\tilde{\mathbf{F}})$. $\tilde{Y}$ is RCLL semimartingale and the stochastic basis $(\Omega, \mathcal{F}, \tilde{\mathbf{F}}, \mathbf{P})$ satisfies the usual conditions. Suppose $\tilde{Y}$ satisfied the conditions NFLVR with $\phi \in \mathcal{P}(\tilde{\mathbf{F}})$ admissible portfolio and $(\phi \cdot Y)_{\infty}$ exits a.s.; then, there is ELMM $\mathbf{Q} \sim \mathbf{P}$ such that $\phi \cdot \tilde{Y}$ is a local martingale, i.e., let $\tilde{\mathcal{F}}_{s}=\mathcal{F}_{s+}^{\mathbf{P}} \in \tilde{\mathbf{F}}$; then, $\phi \cdot \tilde{Y}_{s}=\mathbf{E}_{\mathbf{Q}}\left[\phi \cdot \tilde{Y}_{t} \mid \tilde{\mathcal{F}}_{s}\right]$. Knowing that $\phi \cdot \tilde{Y}_{s}$ is a local martingale under $(\tilde{\mathbf{F}}, \mathbf{Q})$ where $\phi \in \mathcal{P}(\tilde{\mathbf{F}})$ is admissible, then how do we recover $Y$ ? and what is the portfolio $\phi$ in $(\mathbf{F}, \mathbf{Q})$ ? Knowing that $\tilde{Y}_{t}=Y_{t+}$, we are going to employ optional projection of the space $(\Omega, \mathcal{F}, \tilde{\mathbf{F}}, \mathbf{Q})$ on $(\Omega, \mathcal{F}, \mathbf{F}, \mathbf{Q})$ to answer these questions. We begin by showing that

$$
\phi \circ Y_{s}=\mathbf{E}_{\mathbf{Q}}\left[\phi \cdot \tilde{Y}_{t} \mid \mathcal{F}_{s}\right] \text { a.s. } \mathbf{Q},
$$

where "." is the stochastic integral with respect to RCLL semimartingale $\tilde{Y}$ and a predictable integrand and " $O$ " is the stochastic integral with respect to RLL optional semimartingales $Y$ with an optional integrand. Again, we are going to identify $\mathbf{E}_{\mathbf{Q}}$ with $\mathbf{E}$ for brevity.

Theorem 3. Let $\phi \cdot \tilde{Y}_{t}$ be a $\mathbf{Q}$-local martingale. Then, $\phi \circ Y_{s}=\mathbf{E}\left[\phi \cdot \tilde{Y}_{t} \mid \mathcal{F}_{s}\right]$ a.s. Q.

Proof. Let $R_{k} \uparrow \infty$ be a sequence of stopping times in $\tilde{\mathbf{F}}$ where $\phi \cdot \tilde{Y}_{t \wedge R_{k}}$ is a martingale for all $k$ and $\mathbf{E}\left[\phi \cdot \tilde{Y}_{t \wedge R_{k}}\right]<\infty$. Note that $\tilde{Y}_{t}=Y_{t+}$ and $\Delta^{+} Y_{t}=Y_{t+}-Y_{t}$. Then, $\Delta^{+} Y_{t}=Y_{t+}-Y_{t}=\tilde{Y}_{t}-Y_{t}=\tilde{Y}_{t}-\tilde{Y}_{t-}=\Delta \tilde{Y}_{t}$. In addition, $d \tilde{Y}_{t}=d Y_{t}+\Delta \tilde{Y}_{t}=d Y_{t}+\Delta^{+} Y_{t}$ and $\mathbf{E}\left[\phi \cdot \tilde{Y}_{t}-\phi \cdot \tilde{Y}_{s} \mid \mathcal{F}_{s}\right]=\mathbf{E}\left[\mathbf{E}\left[\phi \cdot \tilde{Y}_{t}-\phi \cdot \tilde{Y}_{s} \mid \tilde{\mathcal{F}}_{s}\right] \mid \mathcal{F}_{s}\right]=0$ since $\tilde{\mathcal{F}}_{s} \supseteq \mathcal{F}_{s}$ for all s. Moreover, if $Y_{u}$ is evolving in the interval $[s, t)$, then $\tilde{Y}_{u}$ is evolving in the interval $(s, t]$. Hence,

$$
\begin{aligned}
\mathbf{E}\left[\phi \cdot \tilde{Y}_{t}-\phi \cdot \tilde{Y}_{s} \mid \mathcal{F}_{s}\right] & =\mathbf{E}\left[\mathbf{E}\left[\phi \cdot \tilde{Y}_{t}-\phi \cdot \tilde{Y}_{s} \mid \tilde{\mathcal{F}}_{s}\right] \mid \mathcal{F}_{s}\right] \\
& =\mathbf{E}\left[\int_{(0, t]} \phi_{u-} d \tilde{Y}_{u}-\int_{(0, s]} \phi_{u-} d \tilde{Y}_{u} \mid \mathcal{F}_{s}\right] \\
& =\mathbf{E}\left[\int_{(0, s]} \phi_{u-} d \tilde{Y}_{u}+\int_{(s, t]} \phi_{u-} d \tilde{Y}_{u}-\int_{(0, s]} \phi_{u-} d \tilde{Y}_{u} \mid \mathcal{F}_{s}\right] \\
& =\mathbf{E}\left[\int_{(s, t]} \phi_{u-} d \tilde{Y}_{u} \mid \mathcal{F}_{s}\right]=\mathbf{E}\left[\int_{(s, t]} \phi_{u-}\left(d Y_{u}+\triangle \tilde{Y}_{u}\right) \mid \mathcal{F}_{s}\right] \\
& =\mathbf{E}\left[\int_{(s, t]} \phi_{u-} d Y_{u} \mid \mathcal{F}_{s}\right]+\mathbf{E}\left[\int_{(s, t]} \phi_{u-} \triangle \tilde{Y}_{u} \mid \mathcal{F}_{s}\right] \\
& =\mathbf{E}\left[\int_{(s, t]} \phi_{u-} d Y_{u} \mid \mathcal{F}_{s}\right]+\mathbf{E}\left[\int_{[s, t)} \phi_{v} \triangle^{+} Y_{v} \mid \mathcal{F}_{s}\right] \\
& =\mathbf{E}\left[\int_{(s, t]} \phi_{u-} d Y_{u}+\int_{[s, t)} \phi_{v} \triangle^{+} Y_{v} \mid \mathcal{F}_{s}\right] \\
& =\mathbf{E}\left[\int_{(s, t]} \phi_{u-} d Y_{u}+\int_{[s, t)} \phi_{v} d Y_{v+} \mid \mathcal{F}_{s}\right] \\
& =\mathbf{E}\left[\int_{[s, t]} \phi_{u} d Y_{u} \mid \mathcal{F}_{s}\right]=\mathbf{E}\left[\int_{[0, t]} \phi_{u} d Y_{u} \mid \mathcal{F}_{s}\right]+\mathbf{E}\left[\int_{[0, s]} \phi_{u} d Y_{u} \mid \mathcal{F}_{s}\right] \\
& =\mathbf{E}\left[\phi \cdot Y_{t}-\phi \circ Y_{s} \mid \mathcal{F}_{s}\right]=\mathbf{E}\left[\phi \cdot Y_{t} \mid \mathcal{F}_{s}\right]-\phi \circ Y_{s}=0 \Rightarrow \\
& \quad \mathbf{E}\left[\phi \cdot Y_{t} \mid \mathcal{F}_{s}\right]=\phi \circ Y_{s} .
\end{aligned}
$$


Alternatively, one can use approximation methods of stochastic integrals by sums to arrive to the same result. Thus, in the limit of a partition $\Pi_{n}$ of $[0, T]$ where $T$ is some time horizon and for any $t \in[0, T]$,

$$
\phi_{t} \longleftarrow \phi_{0}+\sum \phi_{t_{i-1}} \mathbf{1}_{] t_{i-1}, t_{i+1}\right]}(t),
$$

where we have chosen the intervals, $\left.] t_{i-1}, t_{i+1}\right]$, for which $\phi_{t_{i-1}}=\phi_{t_{i}}$. Then,

$$
\begin{aligned}
\phi \cdot \tilde{Y} & \longleftarrow \sum \phi_{t_{i-1}}\left(\tilde{Y}_{t_{i+1}}-\tilde{Y}_{t_{i-1}}\right)=\sum \phi_{t_{i-1}}\left(\tilde{Y}_{t_{i+1}}-\tilde{Y}_{t_{i}}+\tilde{Y}_{t_{i}}-\tilde{Y}_{t_{i-1}}\right) \\
& =\sum \phi_{t_{i-1}}\left(\tilde{Y}_{t_{i+1}}-\tilde{Y}_{t_{i}}\right)+\phi_{t_{i-1}}\left(\tilde{Y}_{t_{i}}-\tilde{Y}_{t_{i-1}}\right) \text { using } \phi_{t_{i}}=\phi_{t_{i-1}} \\
& =\sum \phi_{t_{i}}\left(\tilde{Y}_{t_{i+1}}-\tilde{Y}_{t_{i}}\right)+\phi_{t_{i-1}}\left(\tilde{Y}_{t_{i}}-\tilde{Y}_{t_{i-1}}\right) .
\end{aligned}
$$

Note that the stochastic integral "." is defined in the usual sense with respect to $(\Omega, \mathcal{F}, \tilde{\mathbf{F}}, \mathbf{Q})$ and is valid under any refinement of the interval $[0, T]$. This allows using the intervals $\left[t_{i-1}, t_{i+1}\right]$ where, in the limit, the integral is defined. Consider a partition of $[s, t]$. Then, the projection of the integral $\int_{s}^{t} \phi_{u-} d \tilde{Y}_{u}$ on $\mathcal{F}_{s}$ is

$$
\begin{aligned}
\mathbf{E}\left[\int_{s}^{t} \phi_{u-} d \tilde{Y}_{u} \mid \mathcal{F}_{s}\right] & =\mathbf{E}\left[\lim \sum \phi_{t_{i-1}}\left(\tilde{Y}_{t_{i+1}}-\tilde{Y}_{t_{i-1}}\right) \mid \mathcal{F}_{s}\right] \\
& =\lim \mathbf{E}\left[\sum \phi_{t_{i-1}}\left(\tilde{Y}_{t_{i+1}}-\tilde{Y}_{t_{i-1}}\right) \mid \mathcal{F}_{s}\right], \text { by localization and integrability } \\
& =\lim \mathbf{E}\left[\sum \phi_{t_{i-1}}\left(\tilde{Y}_{t_{i+1}}-\tilde{Y}_{t_{i}}\right)+\phi_{t_{i-1}}\left(\tilde{Y}_{t_{i}}-\tilde{Y}_{t_{i-1}}\right) \mid \mathcal{F}_{s}\right] \\
& =\lim \mathbf{E}\left[\sum \phi_{t_{i}}\left(\tilde{Y}_{t_{i+1}}-\tilde{Y}_{t_{i}}\right)+\phi_{t_{i-1}}\left(\tilde{Y}_{t_{i}}-\tilde{Y}_{t_{i-1}}\right) \mid \mathcal{F}_{s}\right], \text { by } \phi_{t_{i-1}}=\phi_{t_{i}} .
\end{aligned}
$$

By properties of conditional expectations and knowing that for any $t_{i}, \mathcal{F}_{t_{i}} \supseteq \mathcal{F}_{s}$ and $\phi_{t}$ is $\mathcal{F}_{t}$-measurable, we get

$$
\mathbf{E}\left[\phi \cdot \tilde{Y}_{t}-\phi \cdot \tilde{Y}_{s} \mid \mathcal{F}_{s}\right]=\mathbf{E}\left[\int_{s}^{t} \phi_{u-} d \tilde{Y}_{u} \mid \mathcal{F}_{s}\right]=\lim \mathbf{E}\left[\sum \phi_{t_{i}}\left(\tilde{Y}_{t_{i+1}}-\tilde{Y}_{t_{i}}\right)+\phi_{t_{i-1}}\left(\tilde{Y}_{t_{i}}-\tilde{Y}_{t_{i-1}}\right) \mid \mathcal{F}_{s}\right]
$$

by localization and integrability, pass the limit through the expectation,

$$
\begin{aligned}
& =\mathbf{E}\left[\lim \sum \phi_{t_{i}}\left(\tilde{Y}_{t_{i+1}}-\tilde{Y}_{t_{i}}\right)+\phi_{t_{i-1}}\left(\tilde{Y}_{t_{i}}-\tilde{Y}_{t_{i-1}}\right) \mid \mathcal{F}_{s}\right] \\
& =\mathbf{E}\left[\lim \sum \mathbf{E}\left[\phi_{t_{i}} \tilde{Y}_{t_{i+1}} \mid \mathcal{F}_{t_{i+1}}\right]-\mathbf{E}\left[\phi_{t_{i}} \tilde{Y}_{t_{i}} \mid \mathcal{F}_{t_{i}}\right]+\mathbf{E}\left[\phi_{t_{i-1}} \tilde{Y}_{t_{i}} \mid \mathcal{F}_{t_{i}}\right]-\mathbf{E}\left[\phi_{t_{i-1}} \tilde{Y}_{t_{i-1}} \mid \mathcal{F}_{t_{i-1}}\right] \mid \mathcal{F}_{s}\right] \\
& =\mathbf{E}\left[\lim \sum \phi_{t_{i}} \mathbf{E}\left[\tilde{Y}_{t_{i+1}} \mid \mathcal{F}_{t_{i+1}}\right]-\phi_{t_{i}} \mathbf{E}\left[\tilde{Y}_{t_{i}} \mid \mathcal{F}_{t_{i}}\right]+\phi_{t_{i-1}} \mathbf{E}\left[\tilde{Y}_{t_{i}} \mid \mathcal{F}_{t_{i}}\right]-\phi_{t_{i-1}} \mathbf{E}\left[\tilde{Y}_{t_{i-1}} \mid \mathcal{F}_{t_{i-1}}\right] \mid \mathcal{F}_{s}\right]
\end{aligned}
$$

by $\tilde{Y}_{u}=Y_{u+}=Y_{u}+\Delta^{+} Y_{u}$

$$
\begin{aligned}
& =\mathbf{E}\left[\lim \sum \phi_{t_{i-1}}\left(Y_{t_{i}}-Y_{t_{i-1}}\right)+\phi_{t_{i}}\left(Y_{t_{i+1}}-Y_{t_{i}}\right)+\phi_{t_{i-1}}\left(\triangle^{+} Y_{t_{i}}-\Delta^{+} Y_{t_{i-1}}\right)+\phi_{t_{i}}\left(\triangle^{+} Y_{t_{i+1}}-\Delta^{+} Y_{t_{i}}\right) \mid \mathcal{F}_{s}\right] \\
& =\mathbf{E}\left[\lim \sum \phi_{t_{i-1}}\left(Y_{t_{i}}-Y_{t_{i-1}}\right)+\phi_{t_{i}}\left(Y_{t_{i+1}}-Y_{t_{i}}\right)+\phi_{t_{i-1}}\left(\triangle^{+} Y_{t_{i}}-\Delta^{+} Y_{t_{i-1}}\right)+\phi_{t_{i}}\left(\triangle^{+} Y_{t_{i+1}}-\triangle^{+} Y_{t_{i}}\right) \mid \mathcal{F}_{s}\right] \\
& =\mathbf{E}\left[\int_{s+}^{t} \phi_{u-} d Y_{u}^{r}+\int_{s}^{t-} \phi_{u} d Y_{u+}^{g} \mid \mathcal{F}_{s}\right] \\
& =\mathbf{E}\left[\int_{0+}^{t} \phi_{u-} d Y_{u}^{r}+\int_{0}^{t-} \phi_{u} d Y_{u+}^{g}-\int_{0+}^{s} \phi_{u-d} d Y_{u}^{r}-\int_{0}^{s-} \phi_{u} d Y_{u+}^{g} \mid \mathcal{F}_{s}\right] \\
& =\mathbf{E}\left[\int_{0}^{t} \phi_{u-d} d \tilde{Y}_{u}-\int_{0+}^{s} \phi_{u-} d Y_{u}^{r}-\int_{0}^{s-} \phi_{u} d Y_{u+}^{g} \mid \mathcal{F}_{s}\right] \\
& =\mathbf{E}\left[\phi \cdot \tilde{Y}_{t}-\phi-\cdot Y_{s}^{r}-\phi \odot Y_{s+}^{g} \mid \mathcal{F}_{s}\right] \\
& =\mathbf{E}\left[\phi \cdot \tilde{Y}_{t}-\phi \circ Y_{s} \mid \mathcal{F}_{s}\right]=\mathbf{E}\left[\phi \cdot \tilde{Y}_{t} \mid \mathcal{F}_{s}\right]-\phi \circ Y_{s}=0 .
\end{aligned}
$$

Therefore, $\mathbf{E}\left[\phi \cdot \tilde{Y}_{t} \mid \mathcal{F}_{s}\right]=\phi \circ Y_{s}=\phi \cdot Y_{s}^{r}+\phi \odot Y_{s+}^{g}$ for any $s \leq t$. Note that, in the limit, $\triangle^{+} Y_{t_{i}}-\Delta^{+} Y_{t_{i-1}}=\triangle^{+} Y_{t_{i+1}}-\Delta^{+} Y_{t_{i}}=0$.

Lemma 9. $\phi \circ Y_{t}$ is a local optional martingale under $\mathbf{Q}$. 
Proof. By the result of the above theorem, for any $u \leq s \leq t, \mathbf{E}\left[\phi \circ Y_{s} \mid \mathcal{F}_{u}\right]=\mathbf{E}\left[\mathbf{E}\left[\phi \cdot \tilde{Y}_{t} \mid \mathcal{F}_{s}\right] \mid \mathcal{F}_{u}\right]=$ $\mathrm{E}\left[\phi \cdot \tilde{Y}_{t} \mid \mathcal{F}_{u}\right]=\phi \circ Y_{u}$.

By the same method, we have established NFLVR in the unusual conditions, it is possible to see that if NA1 is satisfied for $\tilde{Y}$ on $(\Omega, \mathcal{F}, \tilde{\mathbf{F}}, \mathbf{Q})$, then it is satisfied for $Y$ on $(\Omega, \mathcal{F}, \mathbf{F}, \mathbf{Q})$. Therefore, one can rest assured that if NFLVR or NA1 are satisfied for RCLL semimartingales on $(\Omega, \mathcal{F}, \tilde{\mathbf{F}}, \mathbf{P})$, then they must be satisfied for their optional version by optional projection on $(\Omega, \mathcal{F}, \mathbf{F}, \mathbf{P})$. Hence, optional markets are free of arbitrage. The same is true for optional defaultable markets as they are a special case of optional markets, where defaultable assets and cash-flows are optional semimartingales.

In practice, it may be of interest to know how to transform a defaultable process, $Y$, to a local optional martingale with a strictly positive local optional martingale deflator, $Z$. Here, we will demonstrate, with a simple example, a method to derive $Z$;

Example 3. Consider $Y=X+H(X-x)$ as in Equation (1). However, suppose that $X$ and $x$ are continuous local martingales and that $[H, X]=[H, x]=0$ (see Remark 5). We are going to find a strictly positive local optional martingale deflator $Z$ for $Y$. Given that $H=M+\mu$ and knowing that the sum of local optional martingales is a local optional martingale and that the products of $Z$ with continuous local martingales $X$ and $(X-x)$ are local optional martingales, then it suffices to consider finding $Z$ such that $Z H$ is a local optional martingale. To do so, we choose $Z=\mathcal{E}(\theta \circ M)>0$ and find the appropriate conditions that $\theta$ must satisfy so that $\mathrm{ZH}$ is a local martingale. We do this as follows:

$$
\begin{gathered}
\mathcal{E}(\theta \circ M) H=H \mathcal{E}(\theta \circ M) \theta \circ M+\mathcal{E}(\theta \circ M) \circ M+\mathcal{E}(\theta \circ M) \circ \mu+\theta \mathcal{E}(\theta \circ M) \circ[M, M] \Rightarrow \\
\mathcal{E}(\theta \circ M) \circ \mu+\theta \mathcal{E}(\theta \circ M) \circ[M, M]=0 \Rightarrow \\
\theta=-\frac{d \mu}{d[M, M]} .
\end{gathered}
$$

With $\theta=-\frac{d \mu}{d[M, M]}$ and $(1+\theta \Delta M)\left(1+\theta \Delta^{+} M\right)>0$, one can easily see that the process ZY is a local optional martingale.

Remark 5. Implicit in the definition $H Y$ is the fact that $X$ and $x$ are the default-free evolution of the components of the asset $Y$ over its life and $H$ is the default process. This allows us to suppose that $H$ and $(X, x)$ are orthogonal. In reality, $X$ and $x$ can't be observed in isolation of $H$. In defaultable markets, we observe the process $X+H(X-x)$. The above calculation of local martingale deflator assumes knowledge of $x, X$ and $H$, and that they are related in a particular way. This is done to prove that local optional martingale deflators can be constructed for defaultable asset $Y$.

Acknowledgments: We wish to thank reviewers and editors for their comments which have improved this work. This research is supported by Natural Sciences and Engineering Research Council of Canada (NSERC) discovery grant 5901.

Author Contributions: The two authors contribute equally to this article.

Conflicts of Interest: The authors declare no conflict of interest.

\section{Appendix A. Optional Calculus}

This section is based on the following papers Galchuk $(1980,1985)$. Let $\left(\Omega, \mathcal{F}, \mathbf{F}=\left(\mathcal{F}_{t}\right)_{t \geq 0}, \mathbf{P}\right)$, $t \in[0, \infty)$, where $\mathcal{F}_{t} \in \mathbf{F}, \mathcal{F}_{s} \subseteq \mathcal{F}_{t}, s \leq t$, be a complete probability space $-\mathcal{F}$ contains all $\mathbf{P}$ null sets. However, the family $\mathbf{F}$ is not assumed to be complete, right or left continuous. We introduce $\mathcal{O}(\mathbf{F})$ and $\mathcal{P}(\mathbf{F})$ as the optional and predictable $\sigma$-algebras on $(\Omega,[0, \infty))$, respectively. $\mathcal{O}(\mathbf{F})$ is generated by all $\mathbf{F}$ adapted processes whose trajectories are right continuous and have a limit from the left. $\mathcal{P}(\mathbf{F})$ is generated by all $\mathbf{F}$ adapted processes whose trajectories are left continuous and have limits on the right. A random process $X=\left(X_{t}\right), t \in[0, \infty)$ is said to be optional if it is $\mathcal{O}(\mathbf{F})$-measurable. In general, an optional process has right and left limits but is not necessarily right or left continuous in $\mathbf{F}$. A random 
process, $\left(X_{t}\right), t \in[0, \infty)$, is predictable if $X \in \mathcal{P}(\mathbf{F})$. Furthermore, in general, a predictable process has right and left limits but may not necessarily be right or left continuous in $\mathbf{F}$. For either optional or predictable processes, we can define the following processes: $X_{-}=\left(X_{t-}\right)_{t \geq 0}$ and $X_{+}=\left(X_{t+}\right)_{t \geq 0}$, $\triangle X=\left(\triangle X_{t}\right)_{t \geq 0}$ such that $\triangle X_{t}=X_{t}-X_{t-}$ and $\triangle^{+} X=\left(\triangle^{+} X_{t}\right)_{t \geq 0}$ such that $\triangle^{+} X_{t}=X_{t+}-X_{t}$. In addition, a process is strongly predictable, which is $X$ in $\mathcal{P}_{s}(\mathbf{F})$, if $X \in \mathcal{P}(\mathbf{F})$ and $X_{+} \in \mathcal{O}(\mathbf{F})$.

On an unusual stochastic basis, three canonical types of stopping times exist: predictable stopping times, $S \in \mathcal{T}\left(\mathbf{F}_{-}\right)$, are such that $(S \leq t)$ is $\mathcal{F}_{t-}$ measurable for all $t$. Totally inaccessible stopping times, $T \in \mathcal{T}(\mathbf{F})$, are such that $(T \leq t)$ is $\mathcal{F}_{t}$ measurable for all $t$; however, we note that $(T<t)$ is not necessarily $\mathcal{F}_{t}$ measurable since $\mathcal{F}_{t}$ is not right continuous. Finally, totally inaccessible stopping times in the broad sense, $U \in \mathcal{T}\left(\mathbf{F}_{+}\right)$, are such that $(U \leq t)$ is $\mathcal{F}_{t+}$ measurable and, since $\mathcal{F}_{t+}$ is right continuous, $(U<t)$ is also $\mathcal{F}_{t+}$ measurable for or all $t$. A process $X=\left(X_{t}\right)_{t \geq 0}$ belongs to the space $\mathcal{J}_{\text {loc }}$ if there is a localizing sequence of stopping times in the broad sense, $\left(R_{n}\right), n \in \mathbb{N}, R_{n} \in \mathcal{T}\left(\mathbf{F}_{+}\right)$, $R_{n} \uparrow \infty$ a.s. such that $X \mathbf{1}_{\left[0, R_{n}\right]} \in \mathcal{J}$ for all $n$, where $\mathcal{J}$ is a space of processes and $\mathcal{J}_{\text {loc }}$ is an extension of $\mathcal{J}$ by localization.

A process $A=\left(A_{t}\right)_{t \geq 0}$ is increasing if it is non-negative, its trajectories do not decrease and for any $t$, and the random variable $A_{t}$ is $\mathcal{F}_{t}$-measurable. Let $\mathcal{V}^{+}(\mathbf{F}, \mathbf{P})\left(\mathcal{V}^{+}\right.$for short) be the collection of increasing processes. An increasing process $A$ is integrable if $\mathbf{E} A_{\infty}<\infty$ and locally integrable if there is a sequence $\left(R_{n}\right) \subset \mathcal{T}\left(\mathbf{F}_{+}\right), n \in \mathbb{N}, R_{n} \uparrow \infty$ a.s. such that $\mathbf{E} A_{R_{n+}}<\infty$ for all $n \in \mathbb{N}$. The collection of such processes is denoted by $\mathcal{A}^{+}\left(\mathcal{A}_{\text {loc }}^{+}\right.$, respectively). A process $A=\left(A_{t}\right), t \in \mathbb{R}_{+}$, is a finite variation process if it has finite variation on every segment $[0, t], t \in \mathbb{R}_{+}$a.s., that is $\operatorname{Var}(A)_{t}<\infty$, for all $t \in \mathbb{R}_{+}$ a.s., where $\operatorname{Var}(A)_{t}=\sum_{0 \leq s<t}\left|\Delta^{+} A_{s}\right|+\int_{0+}^{t}\left|d A_{s}^{r}\right|$ and $A^{r}$ is a right continuous part of $A$. We shall denote by $\mathcal{V}(\mathbf{F}, \mathbf{P})$ ( $\mathcal{V}$ for short) the set of $\mathbf{F}$ adapted finite variation processes. A process $A=\left(A_{t}\right)_{t \geq 0}$ of finite variation belongs to the space $\mathcal{A}$ of integrable finite variation processes if $\mathbf{E}\left[\operatorname{Var}(A)_{\infty}\right]<\infty$. A process $A=\left(A_{t}\right)_{t \geq 0}$ belongs to $\mathcal{A}_{\text {loc }}$ if there is a sequence $\left(R_{n}\right) \subset \mathcal{T}\left(\mathbf{F}_{+}\right), n \in \mathbb{N}, R_{n} \uparrow \infty$, such that $A \mathbf{1}_{\left[0, R_{n}\right]} \in \mathcal{A}$ for any $n \in \mathbb{N}$, i.e., for any $n, \mathbf{E}\left[\operatorname{Var}(A)_{R_{n}}\right]<\infty$. A finite variation or an increasing process $A$ can be decomposed to $A=A^{r}+A^{g}=A^{c}+A^{d}+A^{g}$, where $A^{c}$ is continuous, $A^{r}$ is a right continuous, $A^{d}$ is discrete right continuous, and $A^{g}$ is discrete left continuous.

A process $M=\left(M_{t}\right)_{t \geq 0}$ is an optional martingale (supermartingale, submartingale) if $M \in \mathcal{O}(\mathbf{F})$, the random variable $M_{T} \mathbf{1}_{T<\infty}$ is integrable for any stopping time $T \in \mathcal{T}(\mathbf{F})$ and there exists an integrable random variable $\mu \in \mathcal{F}_{\infty}$ such that $M_{T}=\mathbf{E}\left[\mu \mid \mathcal{F}_{T}\right]$ (respectively, $M_{T} \geq \mathbf{E}\left[\mu \mid \mathcal{F}_{T}\right]$, $\left.M_{T} \leq \mathbf{E}\left[\mu \mid \mathcal{F}_{T}\right]\right)$ a.s. for any stopping time $T \in \mathcal{F}_{\infty}$ with $(T<\infty)$. Let $\mathcal{M}(\mathbf{F}, \mathbf{P}) \mathcal{M}$ for short denote the set of optional martingales and $\mathcal{M}_{\text {loc }}$ the set of optional local martingales. If $M$ is local optional martingale, then it can be decomposed to $M=M^{r}+M^{g}$, where $M^{r}=M^{c}+M^{d}, M^{c}$ is continuous, $M^{d}$ is right continuous and $M^{g}$ is left continuous local optional martingales. $M^{d}$ and $M^{g}$ are orthogonal to each other and to any continuous (local) martingale.

An optional semimartingale $X=\left(X_{t}\right)_{t \geq 0}$ can be decomposed to an optional local martingale and an optional finite variation processes; $X=X_{0}+M+A$, where $M \in \mathcal{M}_{l o c}$ and $A \in \mathcal{V}$. A semimartingale $X$ is called special if the above decomposition exists with a strongly predictable process $A \in \mathcal{A}_{\text {loc }}$. Let $\mathcal{S}(\mathbf{F}, \mathbf{P})$ denote the set of optional semimartingales and $\mathcal{S} p(\mathbf{F}, \mathbf{P})$ the set of special optional semimartingales. If $X \in \mathcal{S} p(\mathbf{F}, \mathbf{P})$, then the semimartingale decomposition is unique. By optional martingale decomposition and decomposition of predictable processes, we can decompose a semimartingale further to $X=X_{0}+X^{r}+X^{g}$ with $X^{r}=A^{r}+M^{r}, X^{g}=A^{g}+M^{g}$ and $M^{r}=M^{c}+M^{d}$, where $A^{r}$ and $A^{g}$ are finite variation processes that are right and left continuous, respectively $-M^{r} \in \mathcal{M}_{l o c}^{r}$ right continuous local martingales, $M^{d} \in \mathcal{M}_{l o c}^{d}$ discrete right continuous local martingales and $M^{g} \in \mathcal{M}_{l o c}^{g}$ a left continuous local martingales. This decomposition is useful for defining integration with respect to optional semimartingales. A stochastic integral with respect to optional semimartingale was defined as

$$
\varphi \circ X_{t}=\int_{0}^{t} \varphi_{s} d X_{s}=\int_{0+}^{t} \varphi_{s-} d X_{s}^{r}+\int_{0}^{t-} \varphi_{s} d X_{s+}^{g} .
$$


The stochastic integral with respect to the finite variation processes or strongly predictable processes $A^{r}$ over $(0, t]$ and $A^{g}$ over $[0, t)$ are interpreted as usual, in the Lebesgue sense. The integral $\int_{0+}^{t} \varphi_{s-} d M_{s}^{r}$ over $(0, t]$ is our usual stochastic integral with respect to RCLL local martingale, whereas $\int_{0}^{t-} \phi_{s} d M_{s+}^{g}$ over $[0, t)$ is Galchouk stochastic integral with respect to left continuous local martingale. In general, the stochastic integral with respect to optional semimartingale $X$ can be defined as a bilinear form $Y_{t}=(f, g) \circ X_{t}=f \cdot X_{t}^{r}+g \odot X_{t}^{g}$ such that

$$
f \cdot X^{r}=\int_{0+}^{t} f_{s-} d X_{s}^{r}, g \odot X^{g}=\int_{0}^{t-} g_{s} d X_{s+}^{g},
$$

where $Y$ is again an optional semimartingale $f_{-} \in \mathcal{P}(\mathbf{F})$, and $g \in \mathcal{O}(\mathbf{F})$. Note that the stochastic integral over optional semimartingales is defined on a much larger space of integrands, the product space of predictable and optional processes, $\mathcal{P}(\mathbf{F}) \times \mathcal{O}(\mathbf{F})$. The operator " $\mathrm{o}$ " to denote the stochastic optional integral while the operator "." to denote the regular stochastic integral with respect to RCLL semimartingales, and the operator " $\odot$ " for the Galchouk stochastic integral $g \odot X^{8}$ with respect to left continuous semimartingales. For any semimartingales $X$ and $Y$, their quadratic variation is defined as $[X, Y]=\left[X^{r}, Y^{r}\right]+\left[X^{g}, Y^{g}\right]$ where

$$
\left[X^{r}, X^{r}\right]_{t}=\left\langle X^{c}, X^{c}\right\rangle_{t}+\sum_{0<s \leq t}\left(\triangle X_{s}\right)^{2},\left[X^{g}, X^{g}\right]_{t}=\sum_{0 \leq s<t}\left(\triangle^{+} X_{s}\right)^{2} .
$$

\section{References}

Abdelghani, Mohamed Nabeel. 2016. On The General Theory of Optional Stochastic Processes and Financial Markets Modeling. Ph.D. thesis, University of Alberta, Edmonton, AB, Canada.

Abdelghani, Mohamed Nabeel, and Alexander V. Melnikov. 2016. Financial Markets in the Context of the General Theory of Optional Processes. Cham: Springer International Publishing, pp. 519-28.

Abdelghani, Mohamed Nabeel, and Alexander V. Melnikov. 2017a. A comparison theorem for stochastic equations of optional semimartingales. Stochastics and Dynamics 1850029. doi:10.1142/S0219493718500296.

Abdelghani, Mohamed Nabeel, and Alexander V. Melnikov. 2017b. On linear stochastic equations of optional semimartingales and their applications. Statistics \& Probability Letters 125: 207-14.

Artzner, Philippe, and Freddy Delbaen. 1995. Default risk insurance and incomplete markets. Mathematical Finance 5: 187-95.

Belanger, Alain, Steven E. Shreve, and Dennis Wong. $2001 . \quad$ A unified model for credit derivatives. Mathematical Finance. Available online: https://pdfs.semanticscholar.org/646f/77a7d32dd49a6a552dcdd8582829aaf387ec.pdf (accessed on 21 October 2017)

Bielecki, Tomasz R., and Marek Rutkowski. 2002. Credit Risk: Modeling, Valuation and Hedging. Berlin: Springer Science \& Business Media.

Bingham, Nicholas H., and Rüdiger Kiesel. 2013. Risk-Neutral Valuation: Pricing and Hedging of Financial Derivatives. Berlin: Springer Science \& Business Media.

Black, Fischer, and Myron Scholes. 1973. The pricing of options and corporate liabilities. The Journal of Political Economy 81: 637-54.

Cox, John C., and Stephen A. Ross. 1976. The valuation of options for alternative stochastic processes. Journal of Financial Economics 3: 145-66.

Delbaen, Freddy, and Freddy Schachermayer. 2006. The Mathematics of Arbitrage. Berlin: Springer Science \& Business Media.

Dellacherie, Claude. 1975. Seminaire de Probabilites XI (Lecture Notes in Mathematics). Chapter Deux Remarques sur la Separabilite Optionelle; Berlin: Springer, vol. 581, pp. 47-50.

Duffie, Darrel, and Kenneth J. Singleton. 1997. An econometric model of the term structure of interest-rate swap yields. The Journal of Finance 52: 1287-321.

Duffie, Darrel, and Kenneth J. Singleton. 1999. Modeling term structures of defaultable bonds. The Review of Financial Studies 12: 687-720. 
Duffie, Darrel, and Kenneth J. Singleton. 2012. Credit Risk: Pricing, Measurement, and Management. Princeton: Princeton University Press.

ElKaroui, Nicole, Monique Jeanblanc, and Ying Jiao. 2010. What happens after a default: The conditional density approach. Stochastic Processes and Their Applications 120: 1011-32.

Elliott, Robert J., Monique Jeanblanc, and Marc Yor. 2000. On models of default risk. Mathmatical Finance 10: 179-95.

Elliott, Robert J., and Ekkehard Kopp. 1999. Mathematics of Financial Markets. Berlin/Heidelberg and New York: Springer-Verlag.

Fleming, Thomas R., and David P. Harrington. 2011. Counting Processes and Survival Analysis. New York: John Wiley and Sons.

Gal'chuk, Leonid I. 1980. Optional martingales. Mathematics of the USSR-Sbornik 4: 483-521.

Gal'chuk, Leonid I. 1982. Decomposition of optional supermartingales. Mathematics of the USSR-Sbornik 43: $145-58$.

Gal'chuk, Leonid I. 1985. Stochastic integrals with respect to optional semimartingales and random measures. Theory of Probability and its Applications XXIX 1: 93-108.

Geske, Robert. 1977. The valuation of corporate liabilities as compound options. The Journal of Financial and Quantitative Analysis 12: 541-52.

Geske, Robert. 1979. The valuation of compound options. Journal of Financial Economics 7: 63-81.

Geske, Robert, and Herb E. Johnson. 1984. The valuation of corporate liabilities as compound options: A correction. Journal of Financial and Quantitative Analysis 19: 231-32.

Horowitz, Joseph. 1978. Optional supermartingales and the andersen-jessen theorem. Zeitschrift für Wahrscheinlichkeitstheorie und Verwandte Gebiete 43: 263-72.

Jacod, Jean, and Philip Protter. 2010. Risk-neutral compatibility with option prices. Finance and Stochastics 14: 285-315.

Jeanblanc, Monique 2010. Enlargements of Filtrations. Lecture Notes; Jena: University of Jena.

Jeanblanc, Monique, and Lecam Yann. 2008. Reduced form modelling for credit risk. SSRN. doi:10.2139/ssrn.1021545.

Kardaras, Constantinos. 2012. Market viability via absence of arbitrage of the first kind. Finance and Stochastics 16: 651-67.

Lando, David. 1998. On cox processes and credit-risky securities. Review of Derivatives Research 2: 99-120.

Leland, Hayne E. 1994. Corporate debt value, bond covenants, and optimal capital structure. The Journal of Finance 49: 1213-52.

Leland, Hayne E., and Klaus Bjerre Toft . 1996. Optimal capital structure, endogenous bankruptcy, and the term structure of credit spreads. The Journal of Finance 51: 987-1019.

Lenglart, Erik. 1980. Tribus de meyer et thdorie des processus. Lecture Notes in Mathematics 784: 500-46.

Lepingle, Dominique. 1977. Sur la représentation des sa uts des martingales. Lectures Notes on Mathematics 581: 418-34.

Longstaff, Francis A., and Eduardo S. Schwartz. 1995. A simple approach to valuing risky fixed and floating rate debt. The Journal of Finance 50: 789-819.

Martellini, Lionel, Philippe Priaulet, and Stéphane Priaulet. 2003. Fixed-Income Securities: Valuation, Risk Management and Portfolio Strategies. Chichester: John Wiley \& Sons, vol. 237.

Mertens, Jean-Francois. 1972. Theorie des processus stochastiques generaux applications aux surmartingales. Zeitschrift für Wahrscheinlichkeitstheorie und verwandte Gebiete 22: 45-68.

Merton, Robert C. 1974. On the pricing of corporate debt: The risk structure of interest rates. The Journal of Finance 29: 449-70.

Merton, Robert C. 1976. Option pricing when underlying stock returns are discontinuous. Journal of Financial Economics 3: 125-44.

Musiela, Marek, and Marek Rutkowski. 1997. Continuous-time term structure models: Forward measure approach. Finance and Stochastics 1: 261-91.

(C) 2017 by the authors. Licensee MDPI, Basel, Switzerland. This article is an open access article distributed under the terms and conditions of the Creative Commons Attribution (CC BY) license (http:/ / creativecommons.org/licenses/by/4.0/). 